KAIST-TH 01/03, KIAS-P01012

\title{
Probing the messenger of supersymmetry breaking by the muon anomalous magnetic moment
}

\author{
Kiwoon $\mathrm{Choi}^{a}$, Kyuwan Hwang ${ }^{a}$, Sin Kyu Kang ${ }^{b}$, Kang Young Lee ${ }^{b}$, and Wan Young Song ${ }^{a}$ \\ ${ }^{a}$ Department of Physics, Korea Advanced Institute of Science and Technology \\ Taejon 305-701, Korea \\ ${ }^{b}$ School of Physics, Korea Institute for Advanced Study, Seoul 130-012, Korea
}

\begin{abstract}
Motivated by the recently measured muon's anomalous magnetic moment $a_{\mu}$, we examine the supersymmetry contribution to $a_{\mu}$ in various mediation models of supersymmetry breaking which lead to predictive flavor conserving soft parameters at high energy scale. The studied models include dilaton/modulus-mediated models in heterotic string/ $M$ theory, gaugemediated model, no-scale or gaugino-mediated model, and also the minimal and deflected anomaly-mediated models. For each model, the range of $a_{\mu}^{\text {SUSY }}$ allowed by other experimental constraints, e.g. $b \rightarrow s \gamma$ and the collider bounds on superparticle masses, is obtained together with the corresponding parameter region of the model. Gauge-mediated models with low messenger scale can give any $a_{\mu}^{\text {SUSY }}$ within the $2 \sigma$ bound. In many other models, $b \rightarrow s \gamma$ favors $a_{\mu}^{\text {SUSY }}$ smaller than either the $-1 \sigma$ value $\left(26 \times 10^{-10}\right)$ or the central value $\left(42 \times 10^{-10}\right)$.
\end{abstract}




\section{INTRODUCTION}

Weak scale supersymmetry (SUSY) is perhaps the most promising candidate for physics beyond the standard model (SM) [1]. Any realistic supersymmetric model at the weak scale contains explicit but soft SUSY breaking terms which are presumed to originate from some high energy dynamics. If one writes down the most general form of soft terms, it would require too many parameters, e.g. more than 100 even for the minimal supersymmetric standard model (MSSM). Furthermore, for a generic form of soft terms, the superparticle masses should exceed about $10 \mathrm{TeV}$ in order to avoid dangerous flavor changing processes [2]. Such large superpartner masses spoil the natural emergence of the weak scale, and thus the major motivation for supersymmetry also.

In view of these difficulties of generic soft terms, it is quite demanding to have a theory of soft terms leading to a predictive form of flavor conserving soft terms. In fact, the shape of observable soft terms is mainly determined by the mediation mechanism of SUSY breaking, i.e. by the couplings of the SUSY breaking messenger fields to the observable fields, rather than by the SUSY breaking dynamics itself. This is a good news since in many cases the couplings of the messenger fields can be treated in perturbation theory, while the SUSY breaking dynamics involves nonperturbative effects. Therefore once the messegers of SUSY breaking are identified, one can get a well-defined prediction for soft parameters. As long as the predicted soft parameters conserve the flavors, their size can be of order the weak scale. This would allow the prediction to be tested by the future collider experiments and/or the low energy precision experiments. There already exist many interesting proposals for flavor conserving soft parameters, e.g. dilaton/modulus mediation in heterotic string/ $M$ theory [3.4], gauge mediation [5], no-scale [6] or gaugino mediation [7], anomaly mediation [8, 10], and others 11].

Very recently, the BNL experiment E821 has reported a measurement of the muon's anomalous magnetic moment, indicating a $2.6 \sigma$ deviation of $a_{\mu} \equiv\left(g_{\mu}-2\right) / 2$ from the standard model value [12]:

$$
\Delta a_{\mu} \equiv a_{\mu}^{\exp }-a_{\mu}^{\mathrm{SM}}=(42 \pm 16) \times 10^{-10} .
$$

Although can be consistent with the standard model value if one takes other theoretical calculations of the hadronic vacuum polarization [13], this may indeed be a sign of new physics beyond the standard model. In particular, this deviation can easily find its explanation in supersymmetric models through the well-known neutralino-smuon and chargino-sneutrino diagrams [14]. An explicit formula of the SUSY contribution to $a_{\mu}$ is presented for instance in Ref. [15]. The SUSY contribution to $a_{\mu}$ is enhanced as $\tan \beta$ increases, and the charginosneutrino diagram provides a dominant contribution for generic SUSY parameters. In the limit of degenerate superparticle masses, the leading contribution is approximately given by 14]

$$
a_{\mu}^{\mathrm{SUSY}} \approx \frac{\alpha\left(M_{Z}\right)}{8 \pi \sin ^{2} \theta_{W}} \frac{m_{\mu}^{2}}{m_{S}^{2}} \tan \beta\left(1-\frac{4 \alpha}{\pi} \ln \frac{m_{S}}{m_{\mu}}\right),
$$

where $m_{S}$ denotes the superparticle mass in the loop. It has been pointed out already that this new data on $a_{\mu}$ provides useful information on SUSY parameters [16 25], e.g. upper 
bounds on some superparticle masses. It is also noted that much of the parameter space of the minimal anomaly-mediated model can be excluded by the new data when combined with the constraints from $b \rightarrow s \gamma$ [17]. Possible origin of $\Delta a_{\mu}$ other than SUSY is discussed also in Refs. [26].

In this paper, we wish to study the implications of the precisely measured $a_{\mu}$ for various mediation models of SUSY breaking which lead to predictive forms of flavor conserving soft parameters. The models studied here include the dilaton/modulus-mediated model in heterotic string/ $M$ theory, no-scale or gaugino-mediated model, gauge-mediated model, and also the minimal and deflected anomaly-mediated models [8.10]. In the subsequent analysis, we explore the possibility that the deviation (11) is due to the SUSY contribution to $a_{\mu}$ in these models. Throughout the analysis, we will assume that soft parameters (approximately) conserve CP, which may be necessary to avoid a too large neutron electric dipole moment. If one takes (1) as it is, the corresponding $2 \sigma$ bound on $a_{\mu}^{\text {SUSY }}$ would be given by

$$
10 \times 10^{-10}<a_{\mu}^{\mathrm{SUSY}}<74 \times 10^{-10} .
$$

The inclusive $b \rightarrow s \gamma$ process is known to put strong constraints on the MSSM parameter space. The leading SUSY contribution to $b \rightarrow s \gamma$ comes from the charged Higgs boson and chargino mediated diagrams. The charged Higgs boson diagram contributes constructively, while the chargino diagram interferes with the SM amplitude constructively or destructively depending upon the sign of $\mu$. The branching ratio for $b \rightarrow s \gamma$ is obtained by normalizing the hadronic uncertainty with the semileptonic decay rate [27]:

$$
\frac{B r\left(B \rightarrow X_{s} \gamma\right)}{B r\left(B \rightarrow X_{c} e \bar{\nu}\right)}=\frac{\left|V_{t s}^{*} V_{t b}\right|^{2}}{\left|V_{c b}\right|^{2}} \frac{6 \alpha_{\mathrm{em}}}{\pi f(z)}\left(|D|^{2}+A\right) F
$$

where $f(z)=\left(1-8 z+8 z^{3}-z^{4}-12 z^{2} \ln z\right)$ is the phase space factor of the semileptonic decay with $z=m_{c}^{2} / m_{b}^{2}, F=\left[1-8 \alpha_{s}\left(m_{b}\right) / 3 \pi\right] / \kappa(z)$ for the QCD correction factor $\kappa(z) \approx 1-2 \alpha_{s}\left[2.1(1-z)^{2}+1.5\right] / 3 \pi$ for the semileptonic decay, and the term $A$ describes the bremsstrahlung corrections and virtual corrections satisfying the cancellation of the IR divergence 28]. The amplitude $D$ is determined by the Wilson coefficients at $m_{b}$ which can be obtained by the matching condition at the weak scale and the subsequent RG evolution. We perform the matching at the next-to-leading order (NLO) for the SM contribution, while taking the leading order ( $\mathrm{LO}$ ) matching for the MSSM contributions, i.e. the charged Higgs and the chargino contributions. We then perform the RG evolution down to $m_{b}$ at the NLO to find

$$
D=C_{7}^{(0)}\left(m_{b}\right)+\frac{\alpha_{s}\left(m_{b}\right)}{4 \pi}\left(C_{7}^{(1)}\left(m_{b}\right)+\sum_{i=1,8} r_{i} C_{i}^{(0)}\left(m_{b}\right)\right),
$$

where we follow the notation of [27] and $r_{i}$ is quoted in Refs. [28,29]. Combining the recent CLEO [30] and the ALEPH [31] results, one finds the 2 $\sigma$ constraint 29]

$$
2.18 \times 10^{-4}<\operatorname{Br}\left(B \rightarrow X_{s} \gamma\right)<4.10 \times 10^{-4}
$$

which will be used to constrain the parameter space in our analysis.

For $M_{3} M_{2}>0$, the parameter region of $a_{\mu}^{\mathrm{SUSY}}>0$ is constrained by the lower bound on $\operatorname{Br}\left(B \rightarrow X_{s} \gamma\right)$, while that of $a_{\mu}^{\mathrm{SUSY}}<0$ is constrained by the upper bound. In this 
regard, the minimal anomaly mediation model is exceptional since it predicts $M_{3} M_{2}<0$, so $a_{\mu}^{\text {SUSY }}>0$ is constrained by the upperbound on $\operatorname{Br}\left(B \rightarrow X_{s} \gamma\right)$ [17. It is expected that the constraint from the lower bound becomes weaker when the NLO effects are included [34], while the constraint from the upper bound can become even stronger [17].

About the bounds on Higgs boson and superparticle masses, we use the LEP limit $m_{h}>$ $113.5 \mathrm{GeV}$ [32] and $m_{\tilde{\tau}}>72 \mathrm{GeV}$ [33]. The other superparticle mass bounds [33] are satisfied in the allowed region of the Higgs and stau mass limits except for the case of deflected anomaly mediation in which the chargino mass bound $m_{\chi_{1}^{ \pm}}>103 \mathrm{GeV}$ plays an important role.

In Figs. 1-14, we identify the parameter space of the model which can give $a_{\mu}^{\mathrm{SUSY}}$ in the $2 \sigma$ range (3), while taking into account other experimental constraints, e.g. $b \rightarrow s \gamma$ and the collider bounds on superparticle masses. For dilaton/modulus mediation models in heterotic string/ $M$ theory, $b \rightarrow s \gamma$ favors $a_{\mu}^{\text {SUSY }}$ smaller than the $-1 \sigma$ value $\left(26 \times 10^{-10}\right)$. It should be remarked that this constraint is from the lower bound on $\operatorname{Br}\left(B \rightarrow X_{s} \gamma\right)$, so can be relaxed by the NLO SUSY effects [34,35. The no-scale model is similarly (but less) constrained by $b \rightarrow s \gamma$. Gauge mediation models with low messenger scale can give any $a_{\mu}^{\mathrm{SUSY}}$ within the $2 \sigma$ range (3). However models with high messenger scale favor $a_{\mu}^{\mathrm{SUSY}}$ below the central value $\left(42 \times 10^{-10}\right)$. The minimal anomaly mediation is constained by the upper bound on $\operatorname{Br}\left(B \rightarrow X_{s} \gamma\right)$ implying $a_{\mu}^{\mathrm{SUSY}}$ smaller than the central value. When the NLO correction to the charged Higgs contribution is included, $b \rightarrow s \gamma$ contrains $a_{\mu}^{\mathrm{SUSY}}$ more severely [17]. Possible value of $a_{\mu}^{\text {SUSY }}$ in deflected anomaly mediation is severely constrained by the superparticle mass bounds and also $b \rightarrow s \gamma$, but still there is a small parameter region which gives right value of $a_{\mu}^{\mathrm{SUSY}}$.

To set up the notation, let us consider generic low energy interactions of the MSSM fields. They consist of supersymmetric couplings encoded in the superpotential

$$
W=\frac{1}{6} y_{i j k} \Phi_{i} \Phi_{j} \Phi_{k}-\mu H_{1} H_{2}
$$

and also soft supersymmetry breaking terms which can be written as

$$
-\mathcal{L}_{\mathrm{SB}}= \pm \frac{1}{2} M_{a} \lambda_{a} \lambda_{a}+\frac{1}{2} m_{i j}^{2} \phi_{i} \phi_{j}^{*}+\frac{1}{6} A_{i j k} y_{i j k} \phi_{i} \phi_{j} \phi_{k}+B \mu h_{1} h_{2}+\text { h.c. }
$$

where $y_{i j k}$ denote the Yukawa coupling constants for the MSSM superfields $\Phi_{i}$ which include the quark superfields, the lepton superfields, and also the two Higgs doublet superfields $H_{1}$ and $H_{2}$. Here $M_{a}(a=3,2,1)$ stand for the $S U(3) \times S U(2) \times U(1)$ gaugino masses, $m_{i j}^{2}$ are the soft scalar masses of the scalar components $\phi_{i}$ of the MSSM superfields $\Phi_{i}$, and $A_{i j k}$ and $B$ are the trilinear and bilinear coefficients in the scalar potential. To follow up the most frequently used convention for the relative sign of $M_{a}$ and $A_{i j k}$, we use different sign conventions of $M_{a}$ for different models: + for the dilaton/modulus and no-scale (gaugino) mediation models, - for the gauge and anomaly mediation models.

Each mediation mechanism that will be studied in this paper provides a well-defined prediction for $M_{a}, A_{i j k}$ and $m_{i j}^{2}$ at certain high energy messenger scale. The predicted high energy parameters can be transformed to the low energy values through the standard renormalization group $(\mathrm{RG})$ analysis. In this procedure, we assume the minimal particle content in the observable sector, viz. the MSSM particles. If there exist more particles 
with masses between the messenger scale and the weak scale and also with sizable gauge or Yukawa couplings to the MSSM fields, our results would be changed accordingly. We also ignore the effects of small Yukawa couplings of the 1st and 2nd generations in the RG evolution.

The situation for $\mu$ and $B$ is more involved since they depend on the details of how the $\mu$-term is generated as well as on how SUSY breaking is mediated. In the absence of any definite prediction for $\mu$ and $B$, normally one trade $\mu$ and $B$ for $\tan \beta=\left\langle H_{2}\right\rangle /\left\langle H_{1}\right\rangle$ and $M_{Z}$ through the condition of radiative electroweak symmetry breaking, while leaving $\operatorname{sign}(\mu)$ undetermined. Note that $\mu$ and $B$ do not affect the RG running of other soft parameters, which can be assured by the dimensional argument and selection rules.

It has been noted that an extensive region of the soft parameter space gives rise to a scalar potential with a color or charge breaking minimum or a field direction along which the potential is unbounded from below [36 38]. For instance, it turns out that the entire parameter space of the dilaton/modulus mediation in heterotic string theory and also of the no-scale mediation give such a potentially dangerous scalar potential 36.37. In this paper, we do not require that the scalar potential should have a phenomenologically viable global minimum, so the model is allowed as long as the scalar potential has a local minimum with correct low energy phenomenology.

We also do not take into account the cosmological mass density of the lightest superparticle (LSP) in the MSSM sector. There are many different scenarios in which the LSP mass density computed in the framework of $R$ parity conserving MSSM becomes irrelevant, e.g. a late time inflation triggered by an MSSM singlet, $R$-parity violation, or a modulino/gravitino lighter than the LSP.

\section{PROBING THE MESSENGERS OF SUPERSYMMETRY BREAKING}

In this section, we examine the low energy phenomenology of various mediation mechanisms yielding flavor conserving soft parameters. The main purpose is to see which value of $a_{\mu}^{\text {SUSY }}$ can be obtained without any conflict to $b \rightarrow s \gamma$ and the collider bounds on superparticle masses. The models studied here include the dilaton/modulus-mediated model in heterotic string/ $M$ theory, no-scale or gaugino-mediated model, gauge-mediated model, and finally the minimal and deflected anomaly-mediated models. For each mediation model, the

parameter regions allowed by laboratory tests and also the corresponding value of $a_{\mu}^{\mathrm{SUSY}}$ are summarized in Figs. 1-14.

\section{A. Dilaton/modulus mediation in perturbative heterotic string theory}

One possible scheme for flavor-conserving soft parameters is the dilaton/modulus mediation in the framework of weakly coupled heterotic string theory. The Kähler potential and the gauge kinetic function of the four-dimensional effective supergravity are given by

$$
\begin{aligned}
& K=-\ln \left(S+S^{*}\right)-3 \ln \left(T+T^{*}\right)+\left(T+T^{*}\right)^{n_{i}} \Phi_{i} \Phi_{i}^{*} \\
& 4 \pi f_{a}=S
\end{aligned}
$$


where $S$ and $T$ are the dilaton superfield and the overall modulus superfield, respectively, and $n_{i}$ is the modular weight of the chiral matter superfields $\Phi_{i}$. If all the MSSM superfields have the modular weight $n_{i}=-1$, one finds (at the unification scale $M_{\mathrm{GUT}}$ ) [3]

$$
M_{a}=\sqrt{3} M_{\mathrm{aux}}, \quad m_{i j}^{2}=\left|M_{\mathrm{aux}}\right|^{2} \delta_{i j}, \quad A_{i j k}=-\sqrt{3} M_{\mathrm{aux}},
$$

where $M_{\text {aux }}=m_{3 / 2} \sin \theta$ for the Goldstino angle $\theta$ which is defined as $\tan \theta=F_{S} / F_{T}$. Here we assume that $F_{S} / F_{T}$ is real to avoid a too large neutron electric dipole moment. The above relations can receive string-loop or supergravity-loop corrections [39,40] as well as higher order sigma-model corrections [41]. In weakly coupled heterotic string limit, loop corrections are suppressed by $g_{\mathrm{GUT}}^{2} / 8 \pi^{2}$, so can be safely ignored for our purpose. Also at least in orbifold compactification models, there is no sigma-model correction at string tree level.

In fact, the gauge coupling unification scale $M_{\mathrm{GUT}}$ predicted within the weakly coupled heterotic string theory is bigger than the phenomenologically favored value $2 \times 10^{16} \mathrm{GeV}$ by about one order of magnitude. One attractive way to avoid this difficulty is to go to the strong coupling limit [42], i.e. the Horava-Witten heterotic $M$-theory [43], which will be analyzed in the subsequent discussion. Here we simply assume that $M_{\mathrm{GUT}}$ can be lowered down to $2 \times 10^{16} \mathrm{GeV}$ by some stringy effects, while keeping the boundary conditions of (10) valid. Another potential problem of the boundary condition (10) is that the resulting scalar potential has a color or charge breaking minimum or has a field direction which is unbounded from below [37. We do not take this as a serious problem as long as there exists a local minimum of the potential yielding correct low energy phenomenology.

Some phenomenological consequences of (10) has been studied in [44]. Here we perform a detailed numerical analysis of the low energy phenomenology of the boundary condition (10) at $M_{\mathrm{GUT}}$, including the SUSY contributions to $a_{\mu}$ and $b \rightarrow s \gamma$. As usual, we trade $\mu$ and $B$ for $\tan \beta$ and $M_{Z}$. With this prescription, the dilaton/modulus mediation in perturbative heterotic string theory is described by three input parameters,

$$
M_{\text {aux }}, \quad \tan \beta, \quad \operatorname{sign}(\mu) .
$$

The results of our analysis are depicted in Fig. 1 including the contour plot on the plane of $\left(M_{\text {aux }}, \tan \beta\right)$ with $\mu>0$. Fig. 1 shows that $b \rightarrow s \gamma$ favors $a_{\mu}^{\text {SUSY }}$ smaller than the $-1 \sigma$ value $\left(26 \times 10^{-10}\right)$. This constraint from $b \rightarrow s \gamma$ is expected to be relaxed when the NLO SUSY corrections to $\operatorname{Br}\left(B \rightarrow X_{s} \gamma\right)$ are properly taken into account [35].

\section{B. Dilaton/modulus mediation in heterotic $M$ theory}

It has been pointed out by Witten that the correct value of $M_{\mathrm{GUT}}$ can be naturally obtained in compactified heterotic $M$ theory which corresponds to the strong coupling limit of heterotic $E_{8} \times E_{8}$ string theory [42]. At energy scales below the eleven-dimensional Planck scale, the theory is described by an eleven-dimensional supergravity on a manifold with boundary where the two $E_{8}$ gauge multiplets are confined on the two ten-dimensional boundaries [43]. The compactified heterotic $M$ theory involves two geometric moduli, the eleventh length $(\pi \rho)$ and the volume $(V)$ of six dimensional internal space. In four-dimensional 
effective supergravity 4,45$]$, these two moduli define the scalar components of the chiral superfields $S$ and $T$,

$$
\operatorname{Re}(S)=\frac{V}{(4 \pi)^{2 / 3} \kappa^{4 / 3}}, \quad \operatorname{Re}(T)=\frac{V^{1 / 3} \pi \rho}{(4 \pi \gamma)^{1 / 3} \kappa^{2 / 3}},
$$

where $\kappa^{2}$ is the eleven-dimensional gravitational coupling constant and $\gamma=\frac{1}{6} \sum_{I J K} C_{I J K}$ for the intersection numbers $C_{I J K}=\int \omega_{I} \wedge \omega_{J} \wedge \omega_{K}$ of the integer $(1,1)$ cohomology basis $\left\{\omega_{I}\right\}$. Here the superfields $S$ and $T$ are normalized through the periodicity of their axion components: $\operatorname{Im}(S) \equiv \operatorname{Im}(S)+1$ and $\operatorname{Im}(T) \equiv \operatorname{Im}(T)+1$.

Four-dimensional couplings and scales can be expressed in terms of $\operatorname{Re}(S), \operatorname{Re}(T)$ and $\kappa$, yielding the relations 46, 47

$$
\begin{aligned}
& \frac{M_{\mathrm{P}}^{2}}{M_{\mathrm{GUT}}^{2}}=4 \pi \gamma^{1 / 3} \operatorname{Re}(S) \operatorname{Re}(T), \\
& \frac{4 \pi}{g_{\mathrm{GUT}}^{2}}=\operatorname{Re}(S)+\alpha \operatorname{Re}(T),
\end{aligned}
$$

where $M_{\mathrm{P}} \approx 2.5 \times 10^{18} \mathrm{GeV}$ and $g_{\mathrm{GUT}}^{2} \approx 0.5$ are the four-dimensional Planck scale and gauge coupling constant, respectively, and $\alpha$ is a model-dependent (positive) rational number which is generically of order unity. Putting $M_{\mathrm{GUT}} \approx 2 \times 10^{16} \mathrm{GeV}$, one then finds the following vacuum expectation values $(\mathrm{VEVs})$ of moduli in heterotic $M$-theory:

$$
\langle\operatorname{Re}(S)\rangle=\mathcal{O}\left(\frac{4 \pi}{g_{\text {GUT }}^{2}}\right), \quad\langle\operatorname{Re}(T)\rangle=\mathcal{O}\left(\frac{4 \pi}{g_{\text {GUT }}^{2}}\right) .
$$

It has been noted that the four-dimensional effective supergravity of heterotic $M$-theory can be expanded in powers of $1 / \pi\left(S+S^{*}\right)$ and $1 / \pi\left(T+T^{*}\right)$ [4. At leading order in this expansion, the Kähler potential and gauge kinetic function are given by 45.

$$
\begin{aligned}
& K=-\ln \left(S+S^{*}\right)-3 \ln \left(T+T^{*}\right)+\left(\frac{3}{T+T^{*}}+\frac{\alpha}{S+S^{*}}\right) \Phi_{i} \Phi_{i}^{*}, \\
& 4 \pi f_{a}=S+\alpha T .
\end{aligned}
$$

In fact, holomorphy and the axion periodicity implies that any correction to $f_{a}$ is suppressed by $e^{-2 \pi S}$ or $e^{-2 \pi T}$, so absolutely negligible for the moduli VEVs of Eq. (14). The Kähler potential can receive corrections which are higher order in $1 / \pi\left(S+S^{*}\right)$ or $1 / \pi\left(T+T^{*}\right)$. For the moduli VEVs (14), the effects of such higher order corrections are suppressed by $g_{\mathrm{GUT}}^{2} / 8 \pi^{2}$, so can be ignored also for our purpose. With this observation, one finds the following form of soft parameters in heterotic $M$-theory (again at $M_{\mathrm{GUT}}$ ) when SUSY breaking is mediated by the $F$-components of $S$ and $T$,

$$
\begin{aligned}
M_{a}= & \sqrt{3} m_{3 / 2}\left(\frac{1}{1+\epsilon} \sin \theta+\frac{\epsilon}{\sqrt{3}(1+\epsilon)} \cos \theta\right), \\
A_{i j k}= & -\sqrt{3} m_{3 / 2}\left(\frac{3-2 \epsilon}{3+\epsilon} \sin \theta+\frac{\sqrt{3} \epsilon}{3+\epsilon} \cos \theta\right), \\
m_{i j}^{2}= & \left|m_{3 / 2}\right|^{2} \delta_{i j}\left(1-\frac{3}{(3+\epsilon)^{2}}\left\{\epsilon(6+\epsilon) \sin ^{2} \theta\right.\right. \\
& \left.\left.+(3+2 \epsilon) \cos ^{2} \theta-2 \sqrt{3} \epsilon \cos \theta \sin \theta\right\}\right)
\end{aligned}
$$


where $\theta$ is the Goldstino angle and

$$
\epsilon=\alpha\left(T+T^{*}\right) /\left(S+S^{*}\right)
$$

The above results express $M_{a}, A_{i j k}$ and $m_{i j}^{2}$ in terms of three unknown parameters $m_{3 / 2}, \sin \theta$ and $\epsilon$. Once the $\mu$ and $B$ are traded for $\tan \beta$ and $M_{Z}$ through the condition of radiative electroweak symmetry breaking, the dilaton/modulus mediation in heterotic $M$-theory is described by five input parameters,

$$
m_{3 / 2}, \quad \sin \theta, \quad \epsilon, \quad \tan \beta, \quad \operatorname{sign}(\mu),
$$

so not more predictive than the minimal supergravity model for instance. However in heterotic $M$-theory, the value of $\epsilon$ is severely constrained, which allows the results of (16) become more predictive. For instance, the hidden gauge coupling is given by $4 \pi / g_{\mathrm{H}}^{2}=(1-\epsilon) \operatorname{Re}(S)$, so it is required that $0<\epsilon<1$. Inspecting (13), one also finds that $\epsilon$ can not be significantly smaller than the unity.

Here we consider two different values $\epsilon=0.5,0.8$, and examine the allowed value of $a_{\mu}^{\text {SUSY }}$. The results of our analysis are depicted in Figs. 2-5 for $(\epsilon, \tan \beta)=$ $(0.5,10),(0.8,10),(0.5,30),(0.8,30)$. These figures show that $b \rightarrow s \gamma$ favors $a_{\mu}^{\mathrm{SUSY}}$ smaller than the $-1 \sigma$ value $\left(26 \times 10^{-10}\right)$. Again this constraint is expected to be relaxed when the NLO SUSY corrections to $\operatorname{Br}\left(B \rightarrow X_{s} \gamma\right)$ are included. We note that $a_{\mu}^{\text {SUSY }}$ for $\tan \beta \lesssim 10$ is significantly constrained by other laboratory bounds also, e.g. the lightest Higgs mass bound.

\section{No-scale or gaugino mediation}

It has been known for a long time that no-scale supergravity model with non-minimal gauge kinetic function provides an interesting form of flavor conserving soft terms [6]. For instance, one can consider the no-scale Kähler potential together with the simplest nonminimal gauge kinetic functions:

$$
K=-3 \ln \left(T+T^{*}-\Phi_{i} \Phi_{i}^{*}\right), \quad 4 \pi f_{a}=T,
$$

which give rise to

$$
M_{a}=M_{\mathrm{aux}}, \quad m_{i j}^{2}=0, \quad A_{i j k}=0 .
$$

at the messenger scale which is close to the unification scale. Recently it has been noticed that such no-scale boundary condition can naturally emerge in the framework of brane models in which SUSY is broken on a hidden brane in higher dimensional spacetime [7]. The MSSM matter fields are assumed to be confined on a visible brane. However gauge multiplets propagate in bulk and so couple directly to SUSY breaking on hidden brane. Extra-dimensional locality then assures that the soft parameters of the MSSM matter fields vanish, i.e. $m_{i j}^{2}=A_{i j k}=0$, at the compactification scale $M_{c}$ of the extra dimension, while nonzero gaugino masses are allowed, leading to the name of "gaugino mediation" [7].

In gaugino-mediated model, the compactification scale $M_{c}$ is a model-dependent free parameter. If gaugino masses are universal at $M_{c}=M_{\mathrm{GUT}}$, it is rather difficult that the LSP 
is a neutral particle 48,49 . One can avoid this difficulty either by assuming $M_{c}>M_{\mathrm{GUT}}$ or non-universal gaugino masses [48,49]. However a neutral LSP is not mandatory. For instance, a charged LSP is allowed if $R$-parity is broken or the model includes a modulino lighter than the charged LSP. Here we assume that the no-scale boundary condition (19) is given at $M_{\mathrm{GUT}}=2 \times 10^{16} \mathrm{GeV}$ and examine the resulting SUSY contribution to the muon's anomalous magnetic moment. About $\mu$ and $B$, in gaugino-mediated model, it is rather natural that $B=0$ at $M_{\mathrm{GUT}}$. However in generic no-scale supergravity model, $B$ can be a free parameter, and then the no-scale mediation is described by three input parameters,

$$
M_{\text {aux }}, \quad \tan \beta, \quad \operatorname{sign}(\mu) .
$$

The results of our numerical analysis are summarized in Fig. 6 which is somewhat similar to Fig. 1, i.e. the case of dilaton/modulus mediation in heterotic string theory. The analysis of $b \rightarrow s \gamma$ for the no-scale boundary condition (19) has been performed recently in Ref. [50]. It should be remarked also that the scalar potential resulting from (19) has a color or charge breaking minimum or a field direction along which the potential is unbounded from below [36]. As we mentioned, we do not take this as a serious difficulty as long as the potential has a phenomenologically viable local minimum.

\section{Gauge mediation}

The gauge-mediated SUSY breaking (GMSB) models also provide a quite predictive form of flavor conserving soft parameters [5]. In GMSB models, SUSY breaking is transmitted via the SM gauge interactions of $N$ flavors of messenger superfields $\Psi_{i}, \Psi_{i}^{c}$ which form a vectorlike representation of the SM gauge group, e.g. $N(\mathbf{5}+\overline{\mathbf{5}})$ of $S U(5)$. Then the resulting soft terms are determined by the gauge quantum numbers, so automatically conserve the flavors. The messenger fields are coupled to a gauge singlet Goldstino superfield $X$ through the superpotential

$$
W=\lambda_{i} X \Psi_{i} \Psi_{i}^{c}
$$

When $X$ aquires a VEV for both its scalar and $F$ components, the superpotential $W$ induces the messenger spectrum which is not supersymmetric. Integrating out the messenger fields then give rise to the following MSSM soft parameters at the messenger scale $M \approx$ $\lambda_{i}\langle X\rangle$ :

$$
\begin{aligned}
& M_{a}=N \frac{\alpha_{a}(M)}{4 \pi} \Lambda \\
& m_{i j}^{2}=2 N \delta_{i j} \sum_{a} C_{a}^{i}\left(\frac{\alpha_{a}(M)}{4 \pi}\right)^{2} \Lambda^{2}, \\
& A_{i j k}=0
\end{aligned}
$$

where $\alpha_{a}(a=3,2,1)$ are the GUT-normalized gauge coupling constants of $S U(3)_{c} \times$ $S U(2)_{L} \times U(1)_{Y}, C_{a}^{i}$ is the GUT-normalized quadratic Casimir invariant of the matter field $\Phi_{i}$, and $\Lambda \approx\left\langle F_{X}\right\rangle /\langle X\rangle$. 
In fact, the trilinear couplings $A_{i j k}$ at the messenger scale $M$ receive nonzero contribution at two-loop, however we can safely ignore them since they are further suppressed by the loop factor compared to other soft masses with mass dimension one. Again $\mu$ and $B$ can be traded for $\tan \beta$ through the radiative electroweak symmetry breaking. A distinctive feature of GMSB is that a wide range of the messenger scale $M$ is allowed, e.g. from $\Lambda$ to much higher scale around $10^{15} \mathrm{GeV}$. Then the GMSB model is described by five input parameters,

$$
M, \quad \Lambda, \quad \tan \beta, \quad N, \quad \operatorname{sign}(\mu) .
$$

Low energy phenomenology of GMSB models, including $b \rightarrow s \gamma$ and the anomalous muon magnetic moment $a_{\mu}$, has been studied before [51,52]. Here we examine the allowed value of

$a_{\mu}^{\mathrm{SUSY}}$ for the cases of $(N, M)=\left(1,10^{6}\right),\left(1,10^{10}\right),\left(1,10^{15}\right),\left(5,10^{6}\right),\left(5,10^{10}\right),\left(5,10^{15}\right)$, where the messenger scale $M$ is given in the GeV unit. The results for $\mu>0$ are depicted in Figs. 7-12 which show that models with lower $M$ have a better prospect for $a_{\mu}^{\text {SUSY }}$ bigger than the central value $\left(42 \times 10^{-10}\right)$. In particular, gauge-mediated models with $M \sim 10^{6} \mathrm{GeV}$ can give any $a_{\mu}^{\text {SUSY }}$ within the $2 \sigma$ bound (3). For very high $M \sim 10^{15} \mathrm{GeV}, b \rightarrow s \gamma$ constrains $a_{\mu}^{\mathrm{SUSY}}$ as in no-scale or dilaton/modulus mediation model.

\section{E. Minimal anomaly mediation}

Anomaly mediation assumes that SUSY breaking in the hidden sector is transmitted to the MSSM fields only through the auxiliary component $u$ of the off-shell supergravity multiplet. In the Weyl-compensator formulation, $u$ corresponds to the $F$-component of the Weyl compensator superfield $\phi$ in appropriate gauge. The couplings of $\phi$ to generic matter multiplets are determined by the super-Weyl invariance. Therefore at classical level, $\phi$ is coupled to the MSSM fields only through dimensionful (supersymmetric) couplings, e.g the bare $\mu$ parameter or the coefficients of non-renormalizable terms in the superpotential. However quantum radiative effects induce non-trivial scale dependence of dimensionless couplings, so non-trivial couplings of $\phi$ also. Since $M_{a}, A_{i j k}, m_{i j}^{2}$ are all associated with dimensionless supersymmetric couplings, viz. the gauge couplings $g_{a}$ for $M_{a}$, the wave function renormalization factor $Z_{i}$ for $A_{i j k}$ and $m_{i j}^{2}$, these soft parameters are determined entirely by the running behavior of $g_{a}$ and $Z_{i}$ in pure anomaly-mediated scenario. More explicitly, from the super-Weyl invariant effective lagrangian,

$$
\int d^{4} \theta\left(Z_{i}\left(\mu / \sqrt{\phi \phi^{*}}\right) \Phi_{i}^{*} \Phi_{i}+\frac{1}{8} g_{a}^{-2}\left(\mu / \sqrt{\phi \phi^{*}}\right) V^{a} D \bar{D}^{2} D V^{a}+\ldots\right)
$$

one finds the following pure anomaly-mediated soft parameters

$$
\begin{aligned}
& \tilde{M}_{a}=\frac{1}{2} g_{a}^{2}\left(\frac{d g_{a}^{-2}}{d \ln \mu}\right) \frac{F_{\phi}}{\phi}=-\frac{b_{a} \alpha_{a}}{4 \pi} M_{\mathrm{aux}}, \\
& \tilde{A}_{i j k}=-\frac{1}{2}\left(\frac{d \ln Z_{i}}{d \ln \mu}+\frac{d \ln Z_{j}}{d \ln \mu}+\frac{d \ln Z_{k}}{d \ln \mu}\right) \frac{F_{\phi}}{\phi}=\frac{1}{2}\left(\gamma_{i}+\gamma_{j}+\gamma_{k}\right) M_{\mathrm{aux}}, \\
& \tilde{m}_{i j}^{2}=-\frac{1}{4} \delta_{i j}\left(\frac{d^{2} \ln Z_{i}}{d(\ln \mu)^{2}}\right)\left|\frac{F_{\phi}}{\phi}\right|^{2}=-\frac{\dot{\gamma}_{i}}{4}\left|M_{\mathrm{aux}}\right|^{2} \delta_{i j}
\end{aligned}
$$


where $V^{a}$ are the real superfields for gauge multiplets, $b_{a}=(3,-1,-33 / 5)(a=3,2,1)$ are the one-loop beta function coefficients for $S U(3)_{c} \times S U(2)_{L} \times U(1)_{Y}$ in the GUT normalization.

The expressions of pure anomaly-mediated soft parameters are RG-invariant, so are valid at arbitrary energy scale. Therefore the low energy soft parameters are completely fixed by the low energy values of these couplings and an overall scale $M_{\text {aux }}$. However, as can be seen easily, pure anomaly-mediated scenario is simply excluded because it predicts that sleptons have negative mass-squared. So any phenomenologically viable model of anomaly mediation should involve a mechanism to solve the tachyonic slepton problem. One possibility is to introduce a universal positive mass-squared to all soft scalar masses at some high energy scale, e.g. at $M_{\mathrm{GUT}}$, which defines the minimal anomaly-mediated model:

$$
\begin{aligned}
& M_{a}=\tilde{M}_{a}, \quad A_{i j k}=\tilde{A}_{i j k}, \\
& m_{i j}^{2}\left(M_{\mathrm{GUT}}\right)=\tilde{m}_{i j}^{2}\left(M_{\mathrm{GUT}}\right)+m_{0}^{2} \delta_{i j} .
\end{aligned}
$$

After trading $\mu$ and $B$ for $\tan \beta$ and $M_{Z}$, the minimal anomaly mediation can be parameterized by four input parameters,

$$
M_{\mathrm{aux}}, \quad m_{0}, \quad \tan \beta, \quad \operatorname{sign}(\mu) .
$$

Phenomenological aspects of the minimal anomaly-mediated model have been studied in detail in Ref. [53]. It has been noted also that the very recent measurement of the anomalous magnetic moment of the muon disfavors the minimal anomaly-mediated model when combined with the constraint from $b \rightarrow s \gamma$ [17]. Unlike other models, the minimal anomaly mediation model predicts $M_{3} M_{2}<0$, so the parameter region of $a_{\mu}^{\text {SUSY }}>0$ is constrained by the upper bound on $\operatorname{Br}\left(B \rightarrow X_{s} \gamma\right)$.

Our results depicted in Fig. 13 are similar to [17]. However in our case, $b \rightarrow s \gamma$ provides less severe constraint because we use the LO matching condition for the SUSY contributions to $b \rightarrow s \gamma$. Including the NLO charged Higgs contribution [35] makes the constraint from $b \rightarrow s \gamma$ stronger as in Ref. [17].

\section{F. Deflected anomaly mediation}

There is an interesting modification of the pure anomaly mediation which cures the tachyonic slepton with $M_{3} M_{2}>0$ [9,54]. The parameter region of $a_{\mu}^{\text {SUSY }}>0$ in such model may not be severely constrained by $b \rightarrow s \gamma$. The so-called deflected anomaly mediation is a kind of hybrid between anomaly mediation and gauge mediation, but still all SUSY breaking effects originate from $F_{\phi}$. The model contains a light singlet $X$ which describes a flat direction in supersymmetric limit as well as $N$ flavors of gauge-charged messengers $\Psi_{i}, \Psi_{i}^{c}$ which are coupled to $X$ in the superpotential

$$
W=\lambda_{i} X \Psi_{i} \Psi_{i}^{c}
$$

If the VEV of $X$ is determined by the SUSY breaking effects of $F_{\phi}$, not by SUSY conserving dynamics, one has 


$$
\frac{F_{X}}{X}=\rho \frac{F_{\phi}}{\phi}
$$

where $\rho$ depends on the details of how $X$ is stabilized, but $\rho \neq 1$ in general. For instance, in case that $X$ is stabilized by the Coleman-Weinberg mechanism, one finds $\rho=\mathcal{O}\left(1 / 16 \pi^{2}\right)$ [10,54.

At energy scales below $M \approx \lambda_{i}\langle X\rangle$, the heavy thresholds effects of $\Psi_{i}, \Psi_{i}^{c}$ make all soft parameters to leave the RG trajectory of pure anomaly mediation. We then have

$$
\begin{aligned}
& M_{a}(M)=-\left(b_{a}-N(1-\rho)\right) \frac{\alpha(M)}{4 \pi} M_{\mathrm{aux}}, \\
& A_{i j k}(M)=\tilde{A}_{i j k}(M), \\
& m_{i j}^{2}(M)=\tilde{m}_{i j}^{2}(M)-2 N(1-\rho) \delta_{i j} \sum_{a} C_{a}^{i}\left(\frac{\alpha_{a}(M)}{4 \pi}\right)^{2}\left|M_{\mathrm{aux}}\right|^{2},
\end{aligned}
$$

where $b_{a}=(3,-1,-33 / 5)(a=3,2,1)$, and $\tilde{A}_{i j k}, \tilde{m}_{i j}^{2}$ are the pure anomaly-mediated soft parameters in the MSSM framework. Then the deflected anomaly mediation is described by six input parameters,

$$
M_{\text {aux }}, \quad M, \quad \rho, \quad \tan \beta, \quad N, \quad \operatorname{sign}(\mu) .
$$

For numerical analysis, we take $\rho \approx 0$ which corresponds to the case that $X$ is stabilized by the Coleman-Weinberg mechanism [54]. The results for $(N, \tan \beta)=(6,30)$ are depicted in Fig. 14. Possible value of $a_{\mu}^{\mathrm{SUSY}}$ in this model is severely constrained by the chargino, stau, and lightest Higgs mass bounds as well as by $b \rightarrow s \gamma$, however there is still a small parameter region which provides right value of $a_{\mathrm{SUSY}}$.

\section{CONCLUSION}

The recent BNL measurement of the muon's anomalous magnetic moment $a_{\mu} \equiv\left(g_{\mu}-2\right) / 2$ may indeed be a sign of new physics. In this paper, we examined the possibility that the deviation $\Delta a_{\mu}$ from the SM value is due to the SUSY contribution in the framework of various mediation models of SUSY breaking which give rise to predictive flavor conserving soft parameters at high energy scale. The studied models include the dilaton/modulus mediation in heterotic string/ $M$ theory, no-scale or gaugino mediation, gauge mediation, and also the minimal and deflected anomaly mediation models. For each model, we obtain the range of $a_{\mu}^{\text {SUSY }}$ allowed by other laboratory constraints, e.g. $b \rightarrow s \gamma$ and the bounds on superparticle masses, together with the corresponding parameter region of the model.

For dilaton/modulus mediation models in heterotic string/ $M$ theory, the lower bound on $\operatorname{Br}\left(B \rightarrow X_{s} \gamma\right)$ favors $a_{\mu}^{\text {SUSY }}$ smaller than the $-1 \sigma$ bound $\left(26 \times 10^{-10}\right)$. No-scale model is similarly (but less) constrained by $b \rightarrow s \gamma$. Gauge mediation models with low messenger scale can give any $a_{\mu}^{\text {SUSY }}$ within the $2 \sigma$ bound (3). However when the messenger scale is very high, the allowed value of $a_{\mu}^{\text {SUSY }}$ is constrained by $b \rightarrow s \gamma$ as in no-scale or dilaton/modulus medi-

ation models. The possible value of $a_{\mu}^{\mathrm{SUSY}}>0$ in minimal anomaly mediation is constrained to be smaller than the central value $\left(42 \times 10^{-10}\right)$ by the upper bound on $\operatorname{Br}\left(B \rightarrow X_{s} \gamma\right)$. Deflected anomaly mediation is severely constrained by the superparticle mass bounds and 
also by $b \rightarrow s \gamma$, however there is still a small parameter region which gives right value of $a_{\mu}^{\mathrm{SUSY}}$. Our analysis uses the LO matching condition for the SUSY contributions to $b \rightarrow s \gamma$. It is expected that more involved analysis including the NLO SUSY effects [34 makes the constraint from the lower bound on $\operatorname{Br}\left(B \rightarrow X_{s} \gamma\right)$ weaker, while making the constraint from the upper bound stronger.

While this paper is in completion, there appeared several papers which have some overlap with our work. Ref. [17] contains a discussion of the minimal anomaly-mediated model, which agrees with our result, Refs. [18], [19] contain some discussion of gauge-mediated model, and the dilaton-mediated and gauge-mediated models are discussed in Ref. [25] also.

Acknowledgments: We thank Jonathan L. Feng, Konstantin T. Matchev, James D. Wells and Pyungwon Ko for useful discussions. This work is supported by the BK21 project of the Ministry of Education, KRF Grant No. 2000-015-DP0080, KOSEF Grant No. 20001-11100-001-1, and KOSEF through the CHEP of KNU. 


\section{REFERENCES}

[1] For reviews, see H. P. Nilles, Phys. Rep. 150, 1 (1984); H. E. Haber and G. Kane, ibid. 117, 75 (1985).

[2] F. Gabbiani and A. Masiero, Nucl. Phys. B322, 235 (1989); M. Dine, A. Kagan and R. G. Reigh, Phys. Rev D48, 4269 (1993).

[3] V. Kaplunovsky and J. Louis, Phys. Lett. B306, 269 (1993); A. Brignole, L. E. Ibanez and C. Munoz, Nucl. Phys. B422, 125 (1994) [Erratum: B436, 747 (1995)]

[4] K. Choi, H. B. Kim and C. Munoz, Phys. Rev. D57, 7521 (1998); A. Lukas, B. A. Ovrut and D. Waldram, Phys. Rev. D57, 7529 (1998).

[5] M. Dine and A. E. Nelson, Phys. Rev. D48, 1277 (1993); M. Dine, A. E. Nelson, Y. Nir and Y. Shirman, ibid., 53, 2658; for a review, see G. F. Giudice and R. Rattazzi, Phys. Rep. 322, 419 (1999).

[6] For a review, see A. B. Lahanas and D. V. Nanopoulos, Phys. Rep. 145, 1 (1987).

[7] D. E. Kaplan, G. D. Kribs and M. Schmaltz, Phys. Rev D62, 035010 (2000); Z. Chacko, M. Luty, A. E. Nelson and E. Ponton, JHEP 0001, 003 (2000);

[8] L. Randall and R. Sundrum, Nucl. Phys. B557, 79 (1999).

[9] A. Pomarol and R. Rattazzi, JHEP 9905, 013 (1999).

[10] G. F. Giudice, M. A. Luty, H. Murayama and R. Rattazzi, JHEP 9812, 027 (1998); E. Katz, Y. Shadmi and Y. Shirman, JHEP 9908, 015 (1999); Z. Chacko, M. A. Luty, I. Maksymyk, E. Ponton, JHEP 0004, 001 (2000); M. A. Luty and R. Sundrum, Phys. Rev. D62, 035008 (2000); J. A. Bagger, T. Moroi and E. Poppitz, JHEP 0004, 009 (2000).

[11] K. Choi and K. Y. Lee, Phys. Rev. D54, 6591 (1996); T. Kobayashi and K. Yoshioka, Phys. Rev. Lett. 85, 5527 (2000); Z. Chacko and M. A. Luty, hep-ph/0008103.

[12] H. N. Brown et al., hep-ex/0102017.

[13] F. J. Yndurain, hep-ph/0102312.

[14] For recent calculations, see for instance J. L. Lopez, D. V. Nanopoulos and X. Wang, Phys. Rev. D49, 366 (1994); U. Chattopadhyay and P. Nath, Phys. Rev. D53, 1648 (1996); for a recent review, see A. Czarnecki and W. J. Marciano, hep-ph/0010194.

[15] T. Moroi, Phys. Rev. D53, 6565 (1996) [Erratum, D56, 4424 (1997)]; T. Ibrahim and P. Nath, Phys. Rev. D61, 095008 (2000).

[16] L. Everett, G. L. Kane, S. Rigolin and L-T. Wang, hep-ph/0102145.

[17] J. L. Feng and K. T. Matchev, hep-ph/0102146.

[18] U. Chattopadhyay and P. Nath, hep-ph/0102157.

[19] S. Komine, T. Moroi and M. Yamaguchi, hep-ph/0102204.

[20] J. Hisano and K. Tobe, hep-ph/0102315

[21] T. Ibrahim, U. Chattopadhyay and P. Nath, hep-ph/0102324.

[22] J. Ellis, D. V. Nanopoulos and K. A. Olive, hep-ph/0102331.

[23] R. Arnowitt, B. Dutta, B. Hu and Y. Santoso, hep-ph/0102344.

[24] J. E Kim, B. Kyae and H. M. Lee, hep-ph/0103054.

[25] S. P. Martin and J. D. Wells, hep-ph/0103067.

[26] D. Chakraverty, D. Choudhury and A. Datta, hep-ph/0102180; T. Huang, Z. -H. Lin, L. -Y. Shan and X. Zhang, hep-ph/0102193; D. Choudhury, B. Mukhopadhyaya and S. Rakshit, hep-ph/0102199; S. N. Gninenko and N. V. Krasnikov, hep-ph/0102222; K. Cheung, hep-ph/0102238; P. Das, S. K. Rai and S. Raychaudhuri, hep-ph/0102242; T. 
W. Kephart and H. Pas, hep-ph/0102243; E. Ma and M. Raidal, hep-ph/0102255; Z. Xiong and J. M. Yang, hep-ph/0102259; A. Dedes and H. E. Haber, hep-ph/0102297;

Z. Z. Xing, hep-ph/0102304; M. B. Einhorn and J. Wudka, hep-ph/0103034.

[27] A.J. Buras, hep-ph/9806471.

[28] K.G. Chetyrkin, M. Misiak, and M. Münz, Phys. Lett. B 400, 206 (1997).

[29] A.L. Kagan and M. Neubert, Eur. Phys. J. 7, 5 (1999).

[30] CLEO Collaboration, S. Glenn et al., CLEO-CONF 98-17, ICHEP98 1011.

[31] ALEPH Collaboration, R. Barate et al., Phys. Lett. B 429, 169 (1998).

[32] ALEPH collaboration, R. Barate et al., Phys. Lett. B495, 1 (2000) [hep-ex/0011045]; L3 collaboration, M. Acciarri et al., Phys. Lett. B495, 18 (2000) [hep-ex/0011043]; DELPHI collaboration, P. Abreu et al., Phys. Lett. B 499, 23 (2001); OPAL collaboration, G. Abbiendi et al., Phys. Lett. B499, 38 (2001).

[33] LEP SUSY Working Group, ALEPH, DELPHI, L3 and OPAL experiments, note LEPSUSYWG/01-03.1, LEPSUSYWG/99-01.1, LEPSUSYWG/98-04.1.

[34] G. Degrassi, P. Gambino and G. F. Giudice, JHEP 0012, 009 (2000); M. Carena, D. Garcia, U. Nierste and C. Wagner, Phys. Lett. B499, 141 (2001).

[35] K. Choi, K. Hwang and W. Y. Song, in preparation.

[36] J. A. Casas, A. Lleyda and C. Munoz, Nucl. Phys. B471, 3 (1996);

[37] J. A. Casas, A. Lleyda and C. Munoz, Phys. Lett. B380, 59 (1996).

[38] S. A. Abel and C. Savoy, Phys. Lett. B444, 119 (1998); J. A. Casas, A. Ibarra and C. Munoz, Nucl. Phys. B554, 67 (1999).

[39] J. Louis and Y. Nir, Nucl. Phys. B447, 18 (1995).

[40] K. Choi, C. Munoz and J. S. Lee, Phys. Rev. Lett. 80, 3686 (1998).

[41] K. Choi, Z. Phys. C39, 219 (1988); H. B. Kim and C. Munoz, Z. Phys. C75, 367 (1997).

[42] E. Witten, Nucl. Phys. B471, 135 (1996).

[43] P. Horava and E. Witten, Nucl. Phys. B460, 506 (1996); B475, 94 (1996).

[44] R. Barbieri, J. Louis and M. Moretti, Phys. Lett. B312, 451 (1993) [Erratum: B316, 632 (1993)]; J. Lopez, D. Nanopoulos and A. Zichichi, Phys. Lett. B319, 451 (1993).

[45] A. Lukas, B. A. Ovrut and D. Waldram, Nucl. Phys. B532, 43 (1998); P. Nilles, M. Olechowski and M. Yamaguchi, Nucl. Phys. B530, 43 (1998).

[46] T. Banks and M. Dine, Nucl. Phys. B479, 173 (1996).

[47] K. Choi, Phys. Rev. D56, 6588 (1997); K. Choi, H. B. Kim and H. D. Kim, Mod. Phys. Lett. A14, 125 (1999).

[48] M. Schmaltz and W. Skiba, Phys. Rev. D62, 095005 (2000); Phys. Rev. D62, 095004 (2000).

[49] S. Komine and M. Yamaguchi, Phys. Rev. D63, 035005 (2001).

[50] S. Komine, hep-ph/0102030.

[51] S. Dimopoulos, S. Thomas and J. D. Wells, Nucl. Phys. B488, 39 (1997); S. P. Martin and J. D. Wells, Phys. Rev. D59, 035008 (1999).

[52] M. Carena, G. F. Giudice and C. E. M. Wagner, Phys. Lett. B390, 234 (1997); E. Gabrielli and U. Sarid, Phys. Rev. Lett. 79, 4752 (1997); K. T. Mahanthappa and S. Oh, Phys. Rev. D62, 015012 (2000).

[53] T. Gherghetta, G. F. Giudice and J. D. Wells, Nucl. Phys. B559, 27 (1999); J. L. Feng and T. Moroi, Phys. Rev. D61, 095004 (2000).

[54] R. Rattazzi, A. Strumia, J. D. Wells, Nucl. Phys. B576, 3 (2000). 


\section{FIGURES}

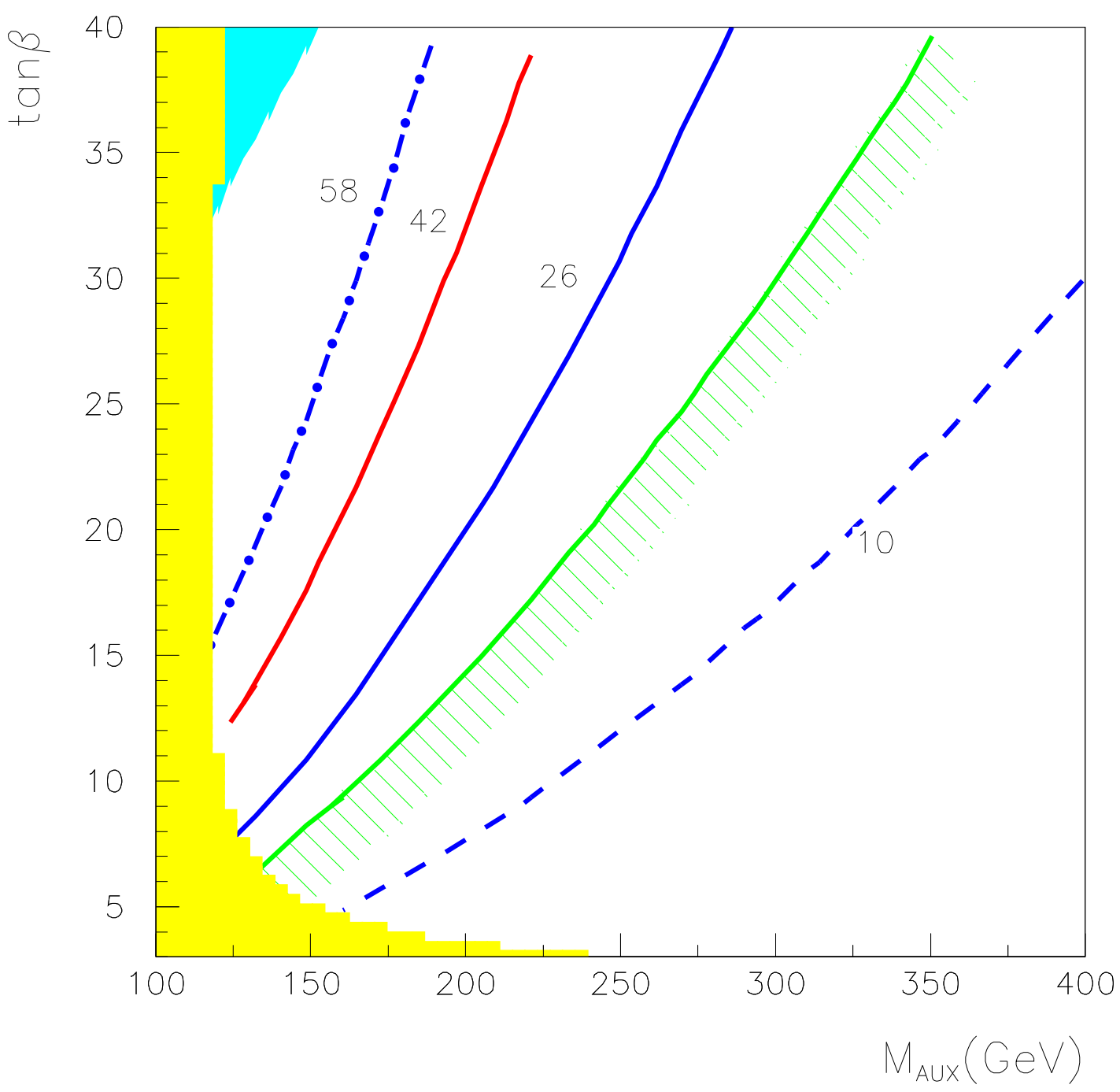

FIG. 1. Contour plot on the plane of $\left(M_{\mathrm{aux}}, \tan \beta\right)$ in the dilaton/modulus mediation model of heterotic string theory. Yellow and cyan regions represent the parameter spaces forbidden by the lightest higgs mass bound and the stau mass bound, respectively. The red line represents the central value of $a_{\mu}^{\mathrm{SUSY}}\left(42 \times 10^{-10}\right)$ and the blue dash-dotted, solid, dashed lines stand for the $+1 \sigma$, $-1 \sigma,-2 \sigma$ values of $a_{\mu}^{\mathrm{SUSY}}$, respectively. The green solid line corresponds to the contour of the $2 \sigma$ lower bound $\operatorname{Br}\left(B \rightarrow X_{s} \gamma\right)=2.18 \times 10^{-4}$ obtained from the SUSY LO calculation and the area below the line is the allowed region. 


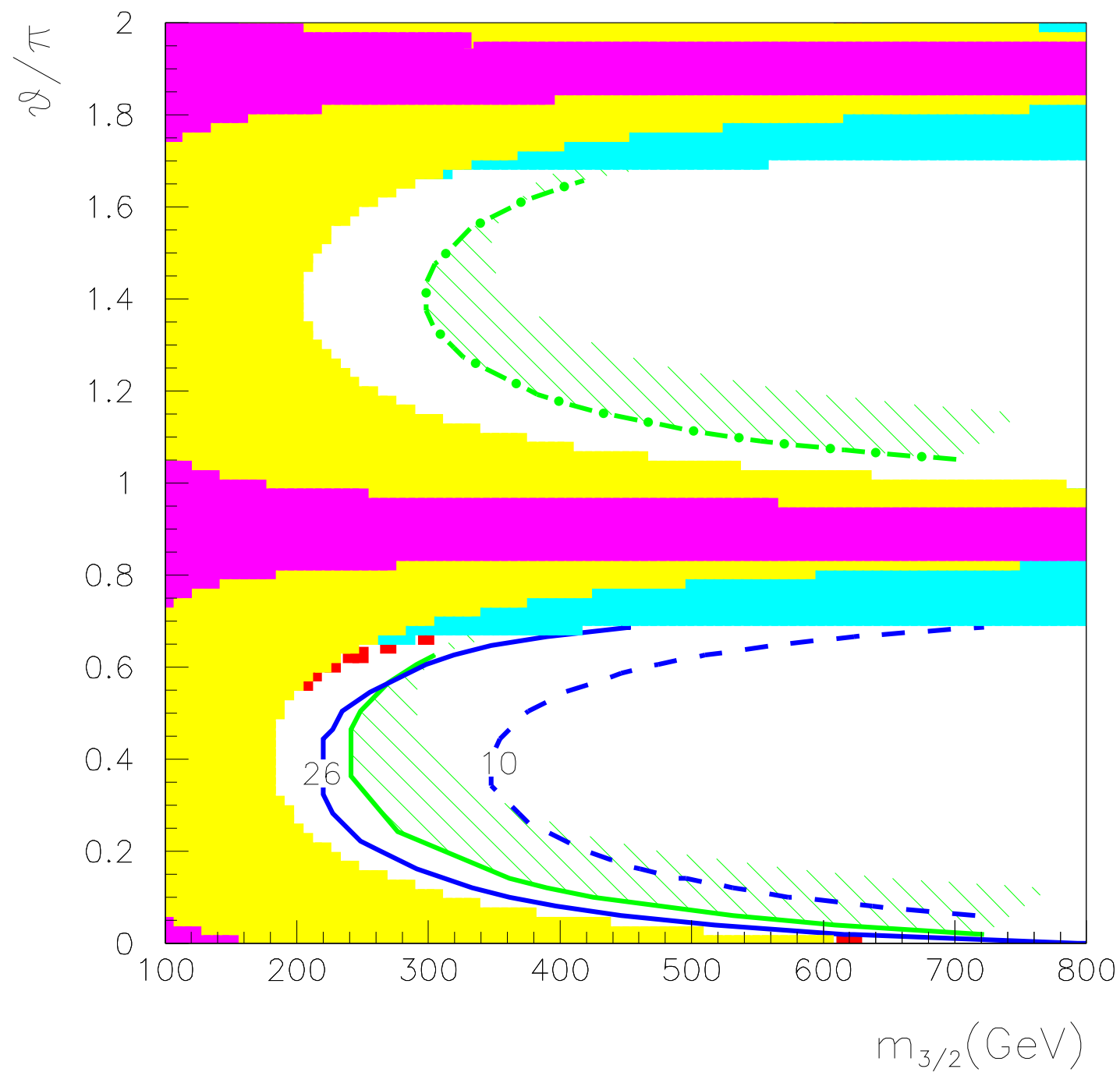

FIG. 2. Contour plot on the plane of $\left(m_{3 / 2}, \theta\right)$ in the dilaton/modulus mediation model of heterotic $M$ theory with $\epsilon=0.5$ and $\tan \beta=10$. Yello, purple and cyan regions represent the parameter space forbidden by the lightest higgs mass bound, the electroweak symmetry breaking condition, and the stau mass bound, respectively. The blue solid(dashed) line is for the $-1 \sigma(-2 \sigma)$ value of $a_{\mu}^{\mathrm{SUSY}}$ and the red colored region represents the area in which $a_{\mu}^{\mathrm{SUSY}}$ is bigger than the central value $\left(42 \times 10^{-10}\right)$. For $\theta>\pi, a_{\mu}^{\text {SUSY }}$ is negative due to the sign flip of the gaugino masses. The green solid and green dash-dotted lines stand for the $2 \sigma$ lower and upper bounds on $\operatorname{Br}\left(B \rightarrow X_{s} \gamma\right)$ and the hashed sides of the lines are the allowed region. 


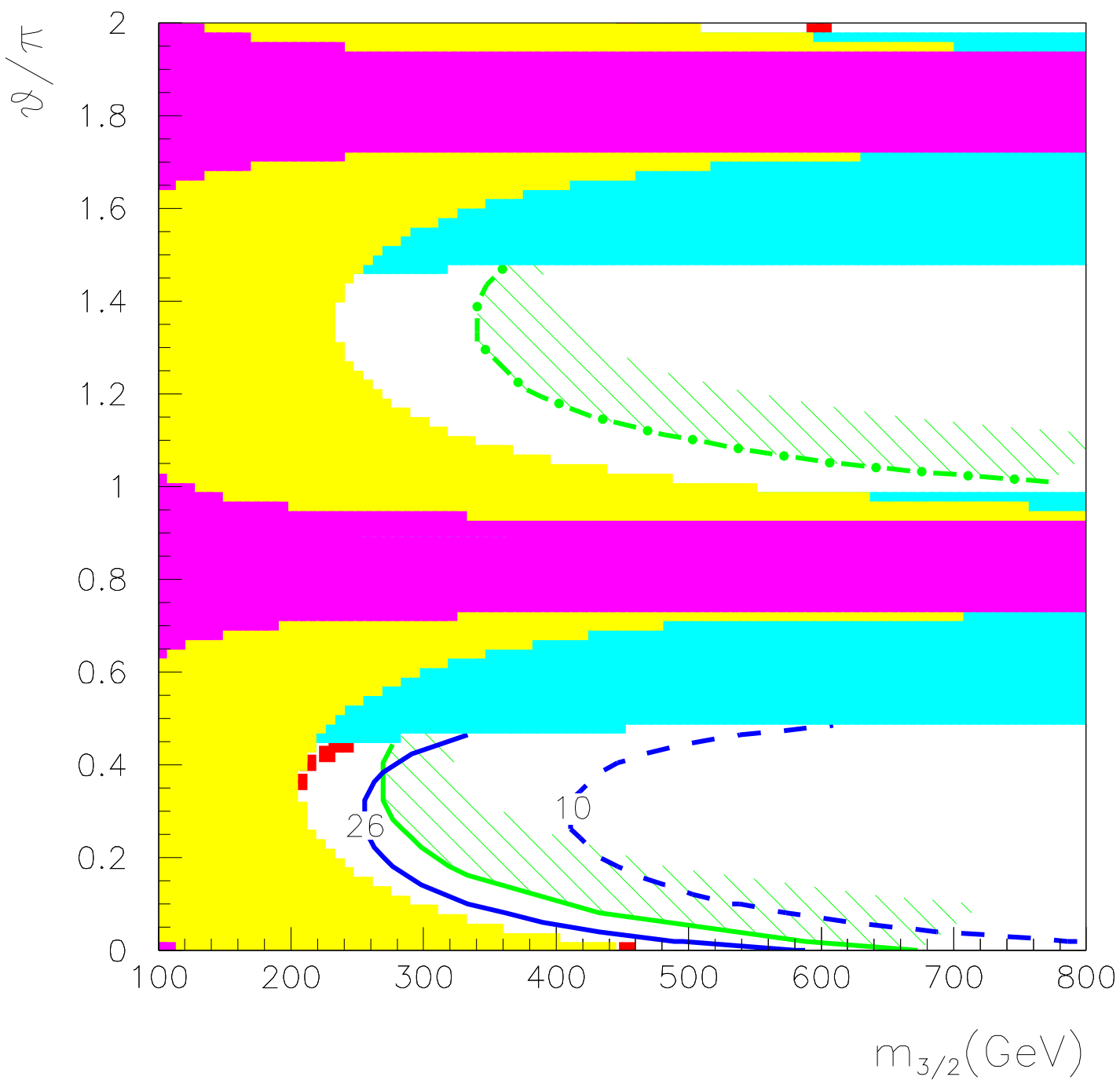

FIG. 3. Contour plot on the plane of $\left(m_{3 / 2}, \theta\right)$ in the dilaton/modulus mediation model of heterotic $M$ theory with $\epsilon=0.8$ and $\tan \beta=10$. 


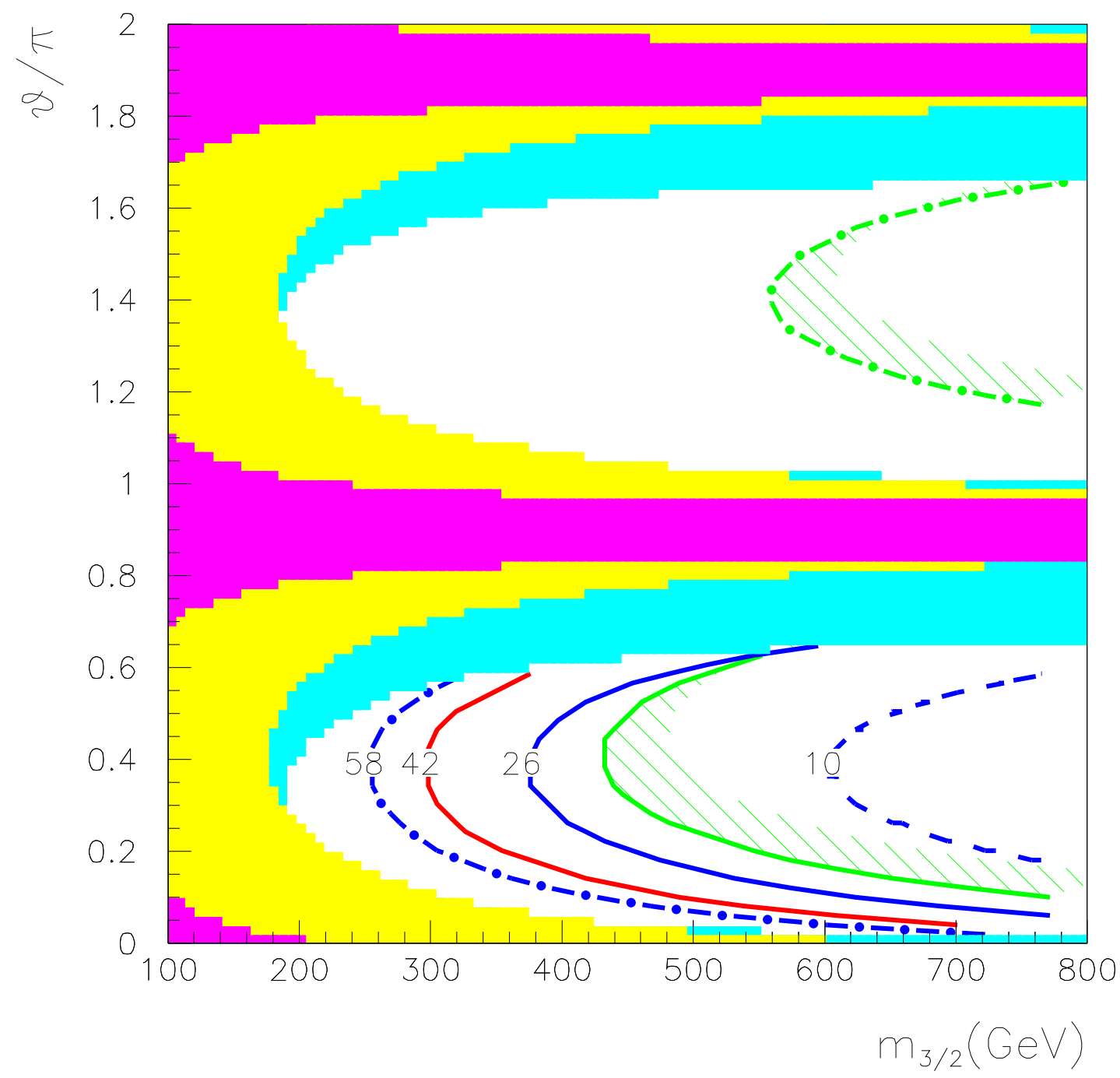

FIG. 4. Contour plot on the plane of $\left(m_{3 / 2}, \theta\right)$ in the dilaton/modulus mediation model of heterotic $M$ theory with $\epsilon=0.5$ and $\tan \beta=30$. The red line is for the centeral value of $a_{\mu}^{\text {SUSY }}$, and the blue dash-dotted, solid, dashed lines stand for the $+1 \sigma,-1 \sigma,-2 \sigma$ values of $a_{\mu}^{\text {SUSY }}$, respectively. 


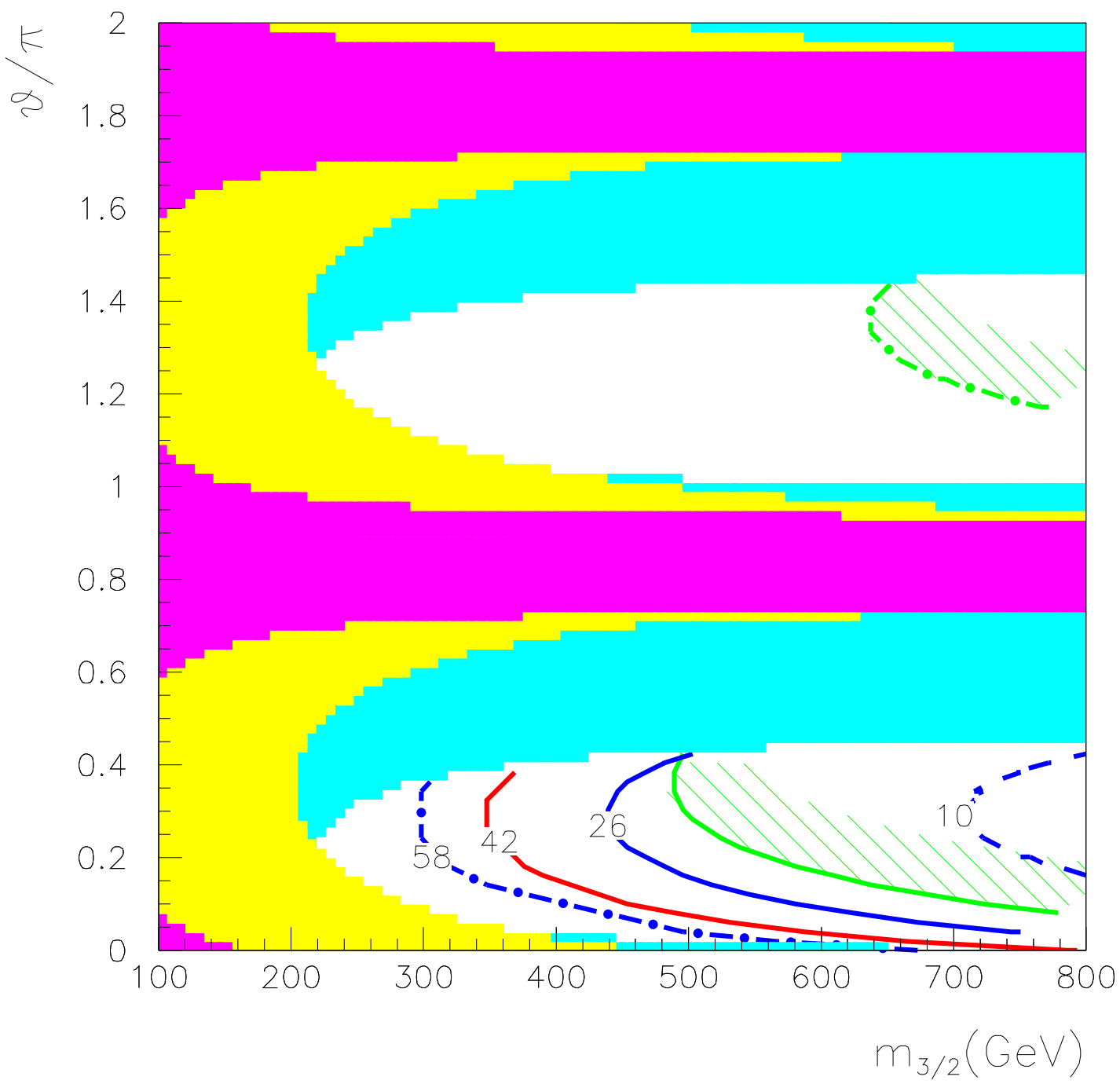

FIG. 5. Contour plot on the plane of $\left(m_{3 / 2}, \theta\right)$ in the dilaton/modulus mediation model of heterotic $M$ theory with $\epsilon=0.8$ and $\tan \beta=30$. 


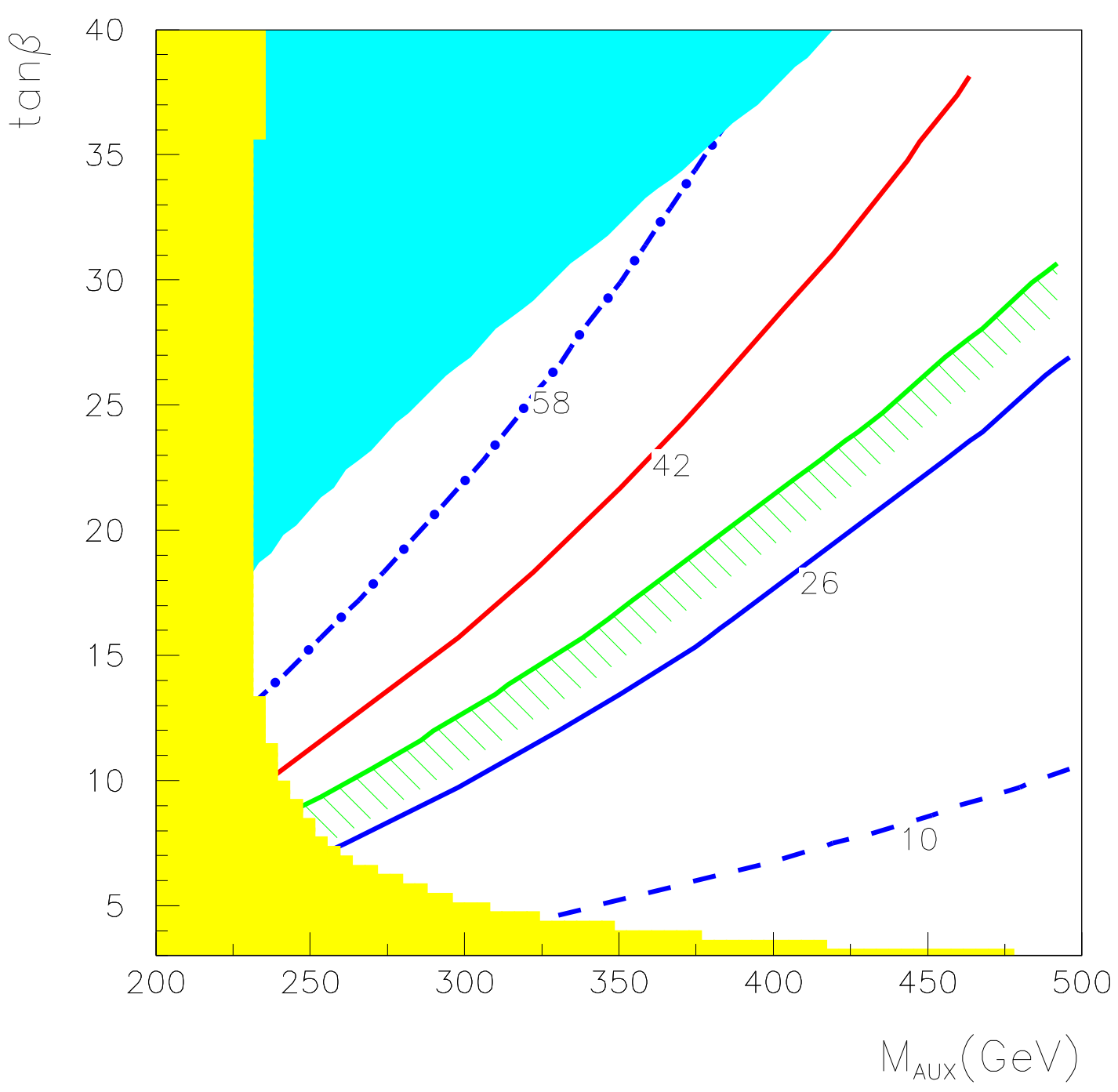

FIG. 6. Contour plot on the plane of $\left(M_{\text {aux }}, \tan \beta\right)$ in no-scale model. Yellow and cyan regions represent the parameter space forbidden by the lightest higgs mass bound and the stau mass bound, respectively. The red line is for the centeral value of $a_{\mu}^{\mathrm{SUSY}}\left(42 \times 10^{-10}\right)$ and the blue dash-dotted, solid, dashed lines stand for the $+1 \sigma,-1 \sigma,-2 \sigma$ values of $a_{\mu}^{\mathrm{SUSY}}$, respectively. The green solid line corresponds to the contour of the $2 \sigma$ lower bound $\operatorname{Br}\left(B \rightarrow X_{s} \gamma\right)=2.18 \times 10^{-4}$ and the area below the line is the allowed region. 


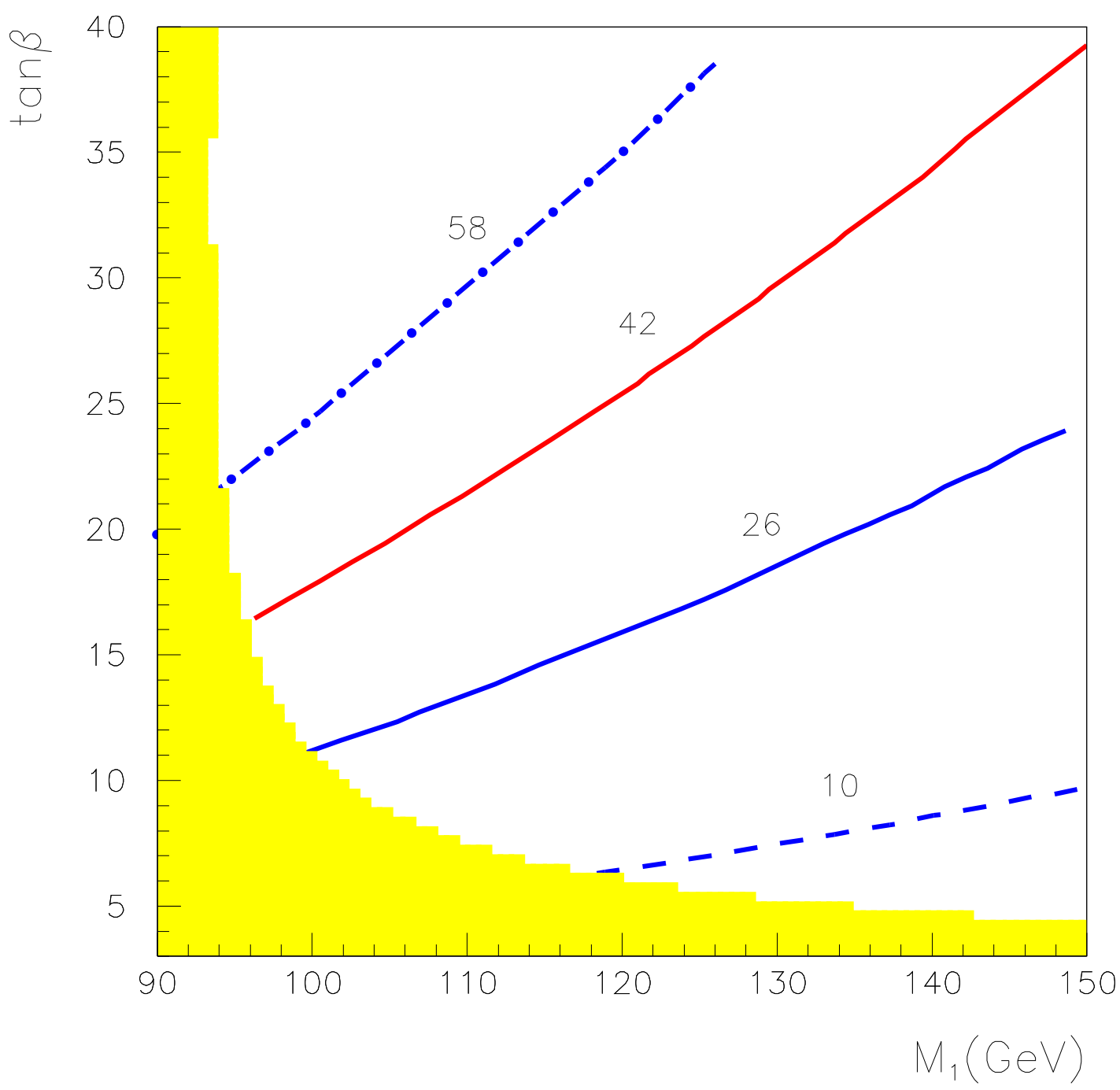

FIG. 7. Contour plot on the plane of $\left(M_{1}, \tan \beta\right)$ in the gauge-mediated supersymmetry breaking (GMSB) model with $N=1$ and $M=10^{6} \mathrm{GeV}$. Yellow region represents the parameter space forbidden by the lightest higgs mass bound. The red line is for the centeral value of $a_{\mu}^{\mathrm{SUSY}}$ $\left(42 \times 10^{-10}\right)$ and the blue dash-dotted, solid, dashed lines stand for the $+1 \sigma,-1 \sigma,-2 \sigma$ values of $a_{\mu}^{\text {SUSY }}$, respectively. The whole parameter space shown in this figure is allowed by the constraints of $\operatorname{Br}\left(B \rightarrow X_{s} \gamma\right)$. 


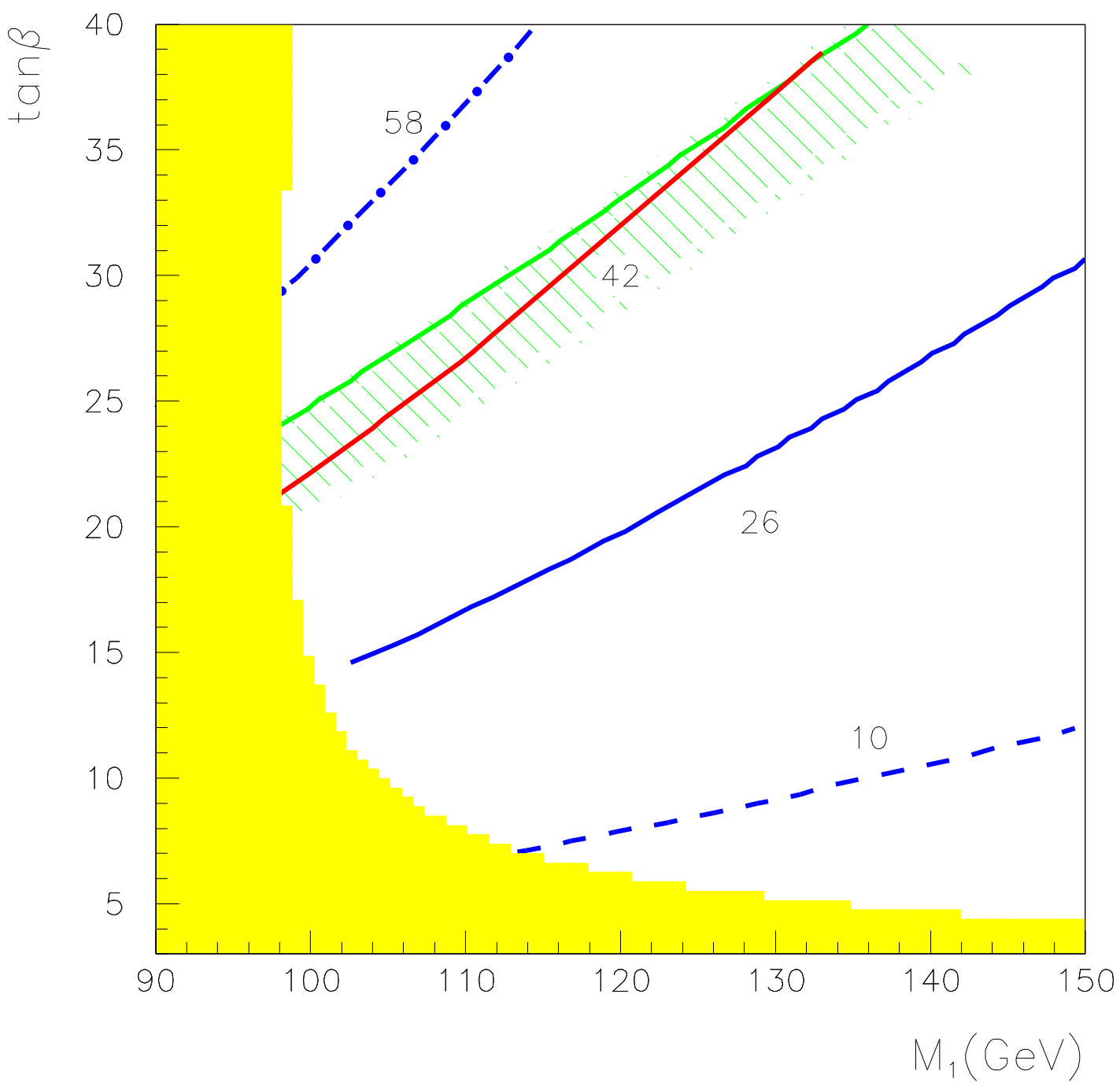

FIG. 8. Contour plot on the plane of $\left(M_{1}, \tan \beta\right)$ in the GMSB model with $N=1$ and $M=10^{10} \mathrm{GeV}$. The green solid line corresponds to the contour of the $2 \sigma$ lower bound $\operatorname{Br}\left(B \rightarrow X_{s} \gamma\right)=2.18 \times 10^{-4}$ and the area below the line is the allowed region. 


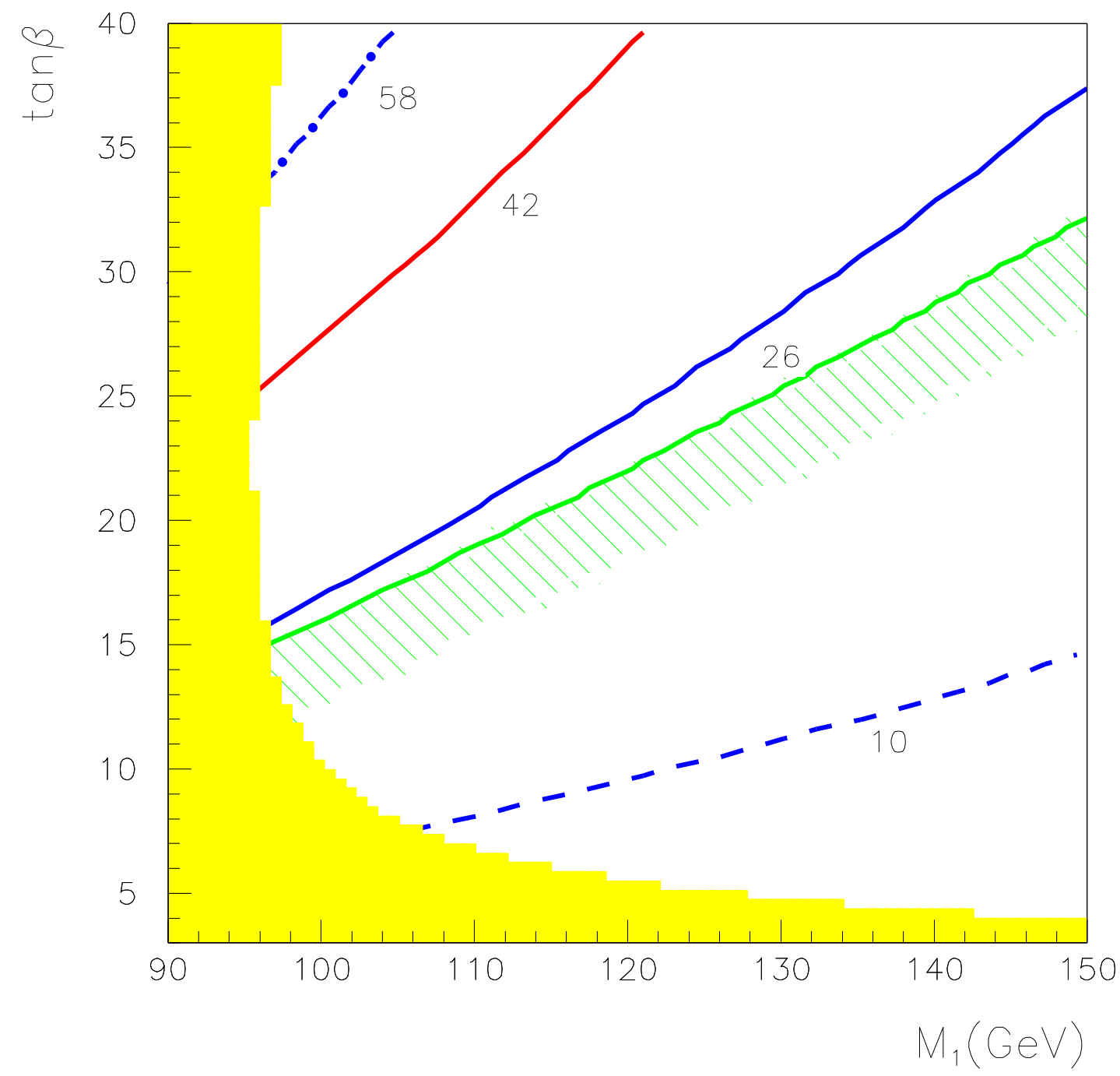

FIG. 9. Contour plot on the plane of $\left(M_{1}, \tan \beta\right)$ in the GMSB model with $N=1$ and $M=10^{15}$ GeV. 


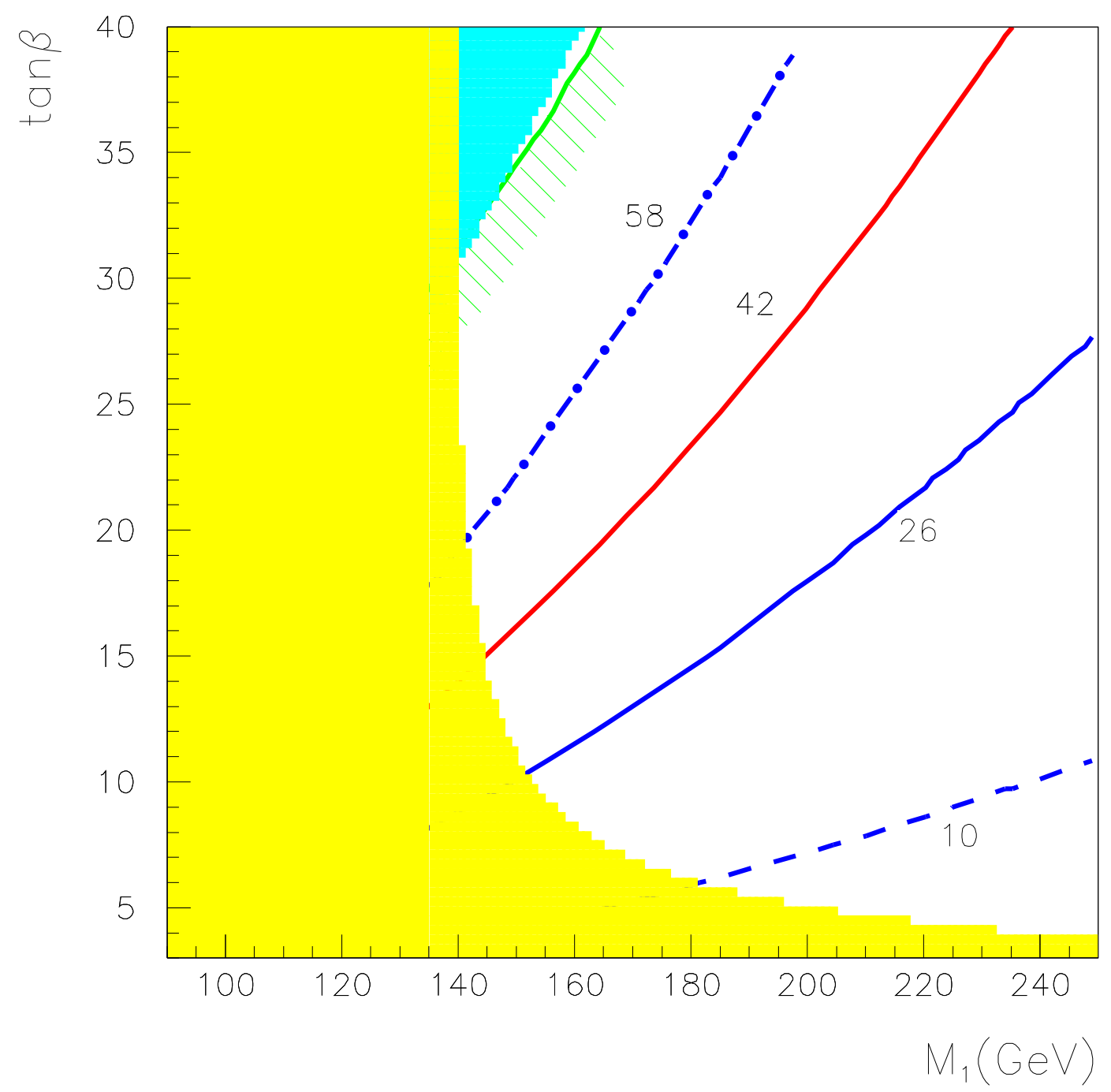

FIG. 10. Contour plot on the plane of $\left(M_{1}, \tan \beta\right)$ in the GMSB model with $N=5$ and $M=10^{6}$ GeV. 


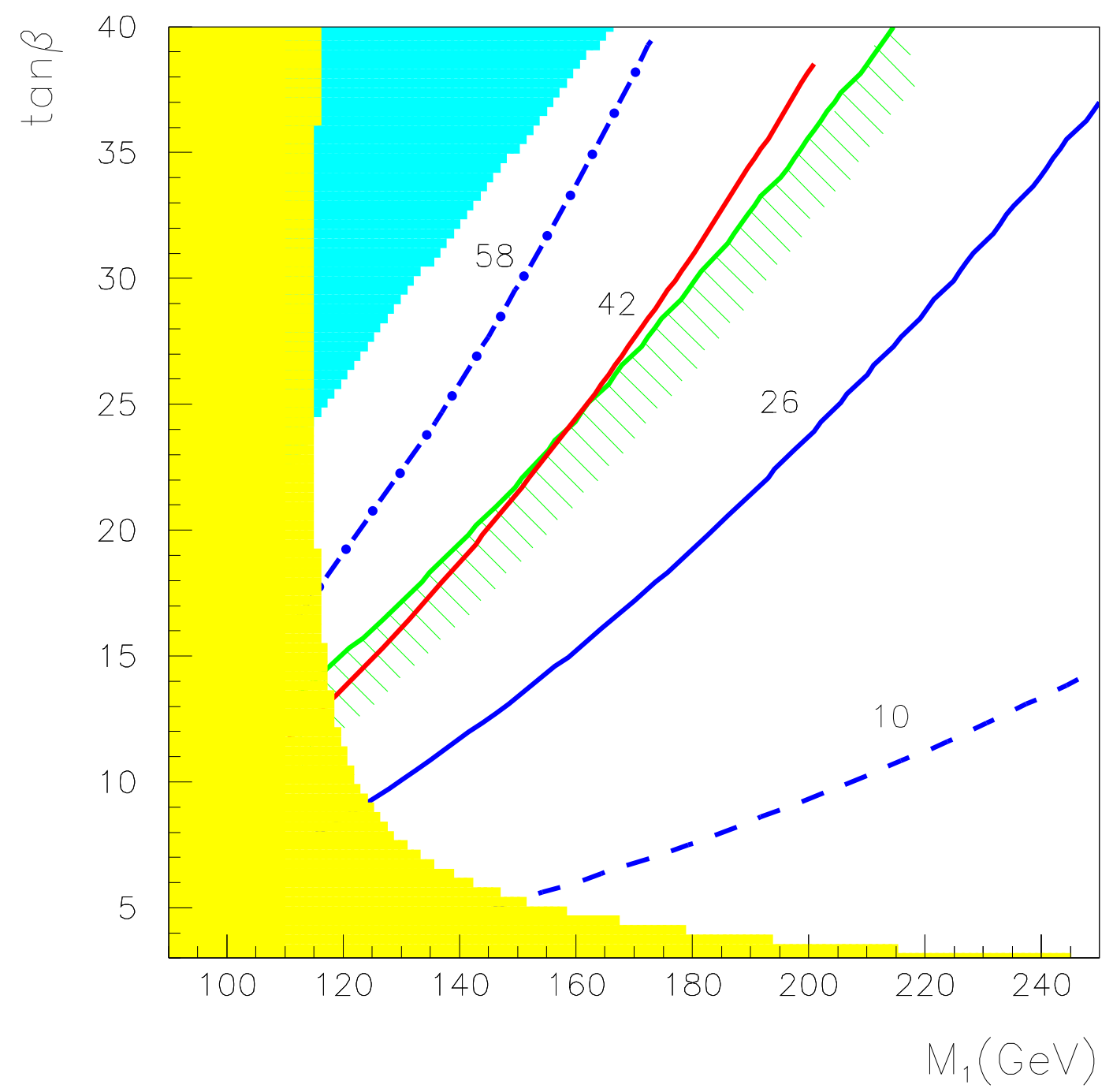

FIG. 11. Contour plot on the plane of $\left(M_{1}, \tan \beta\right)$ in the GMSB model with $N=5$ and $M=10^{10} \mathrm{GeV}$. 


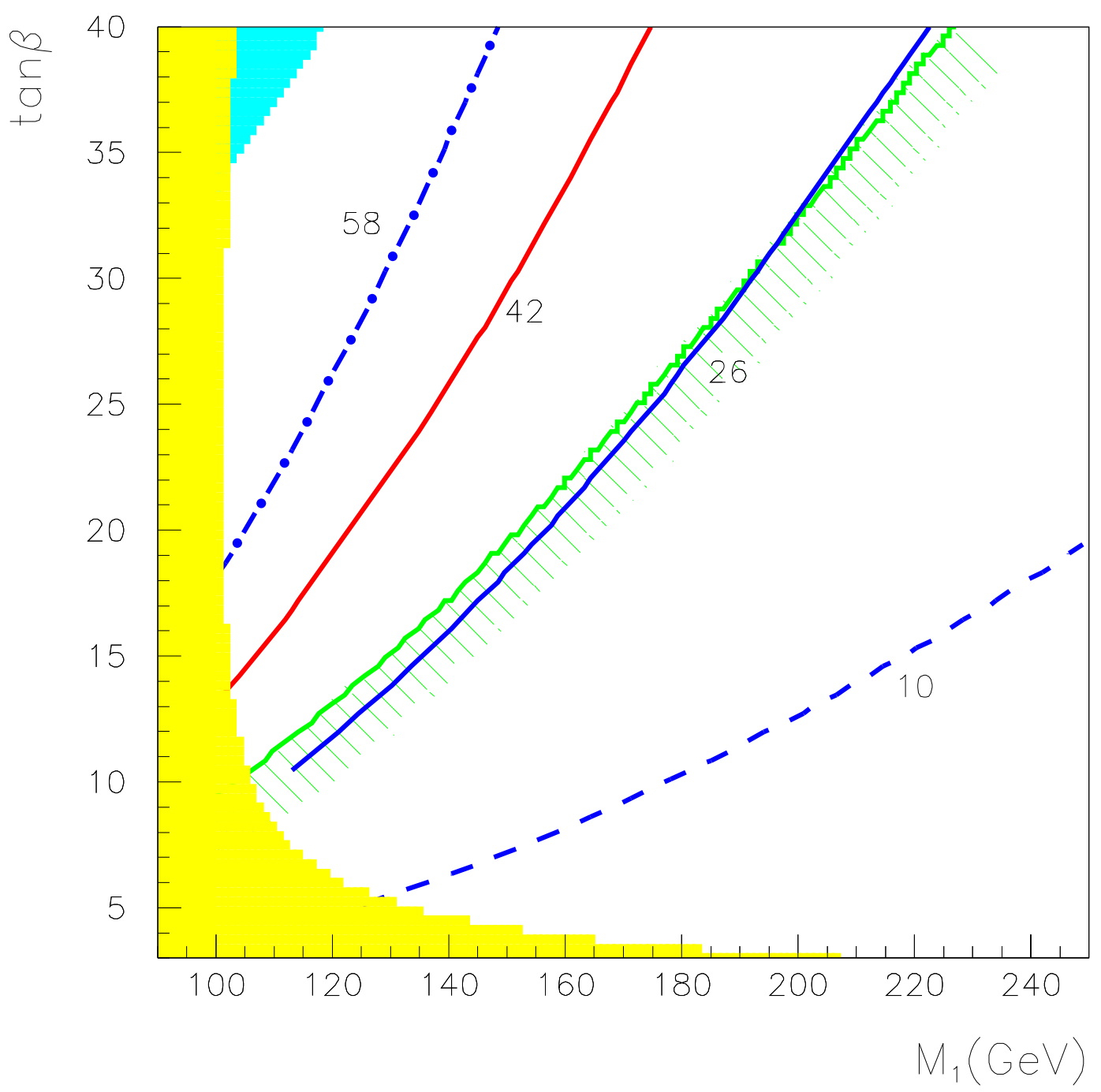

FIG. 12. Contour plot on the plane of $\left(M_{1}, \tan \beta\right)$ in the GMSB model with $N=5$ and $M=10^{15} \mathrm{GeV}$. 


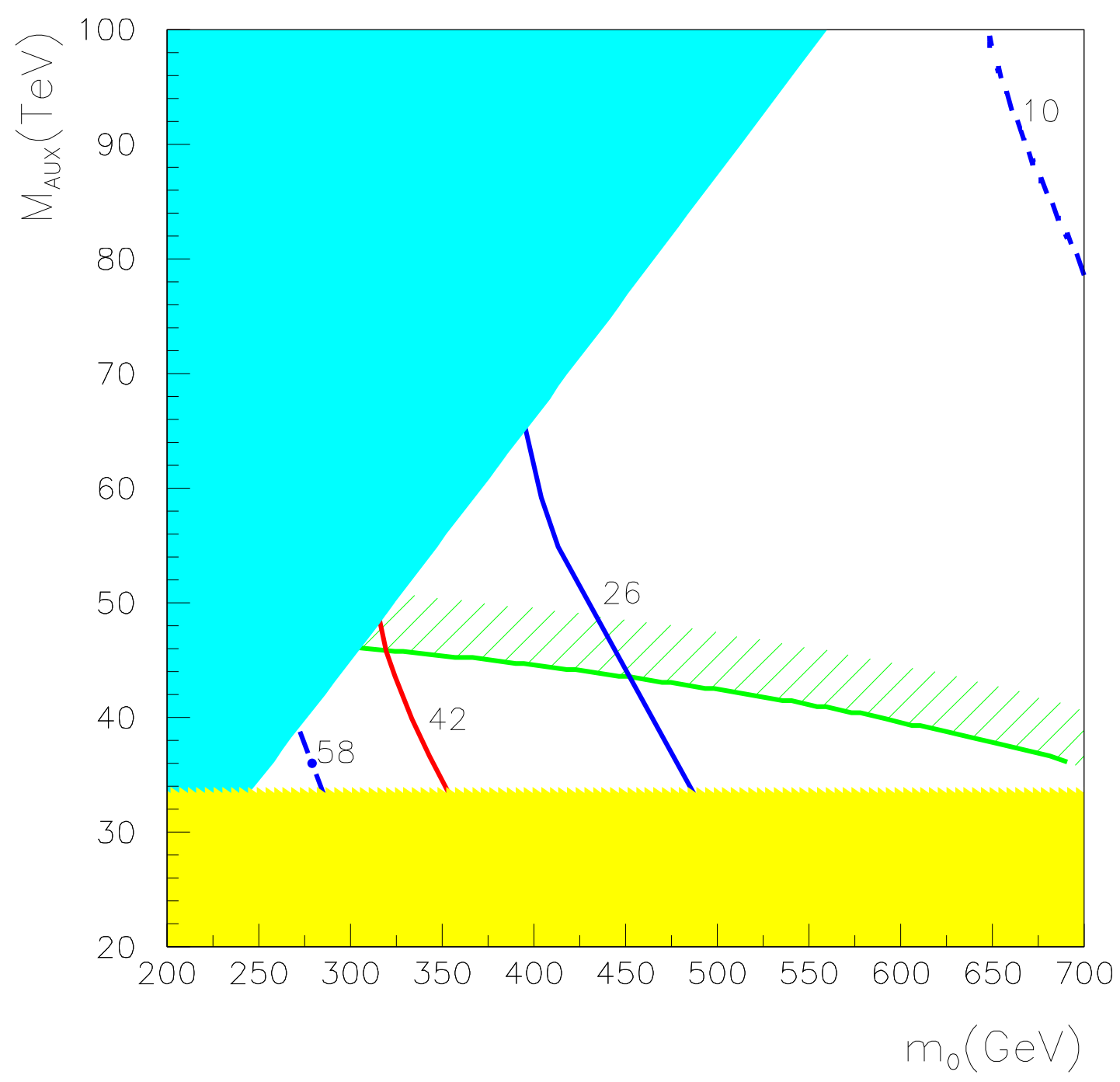

FIG. 13. Contour plot on the plane of $\left(m_{0}, M_{\text {aux }}\right)$ in the minimal anomaly-mediated model with $\tan \beta=30$. Yellow and cyan regions represent the parameter space forbidden by the chargino and stau mass bounds, respectively. The red line is for the centeral value of $a_{\mu}^{\text {SUSY }}\left(42 \times 10^{-10}\right)$ and the blue dash-dotted, solid, dashed lines stand for the $+1 \sigma,-1 \sigma,-2 \sigma$ values of $a_{\mu}^{\text {SUSY }}$, respectively. The green solid line corresponds to the contour of the $2 \sigma$ lower bound $\operatorname{Br}\left(B \rightarrow X_{s} \gamma\right)=2.18 \times 10^{-4}$ and the area above the line is the allowed region. 


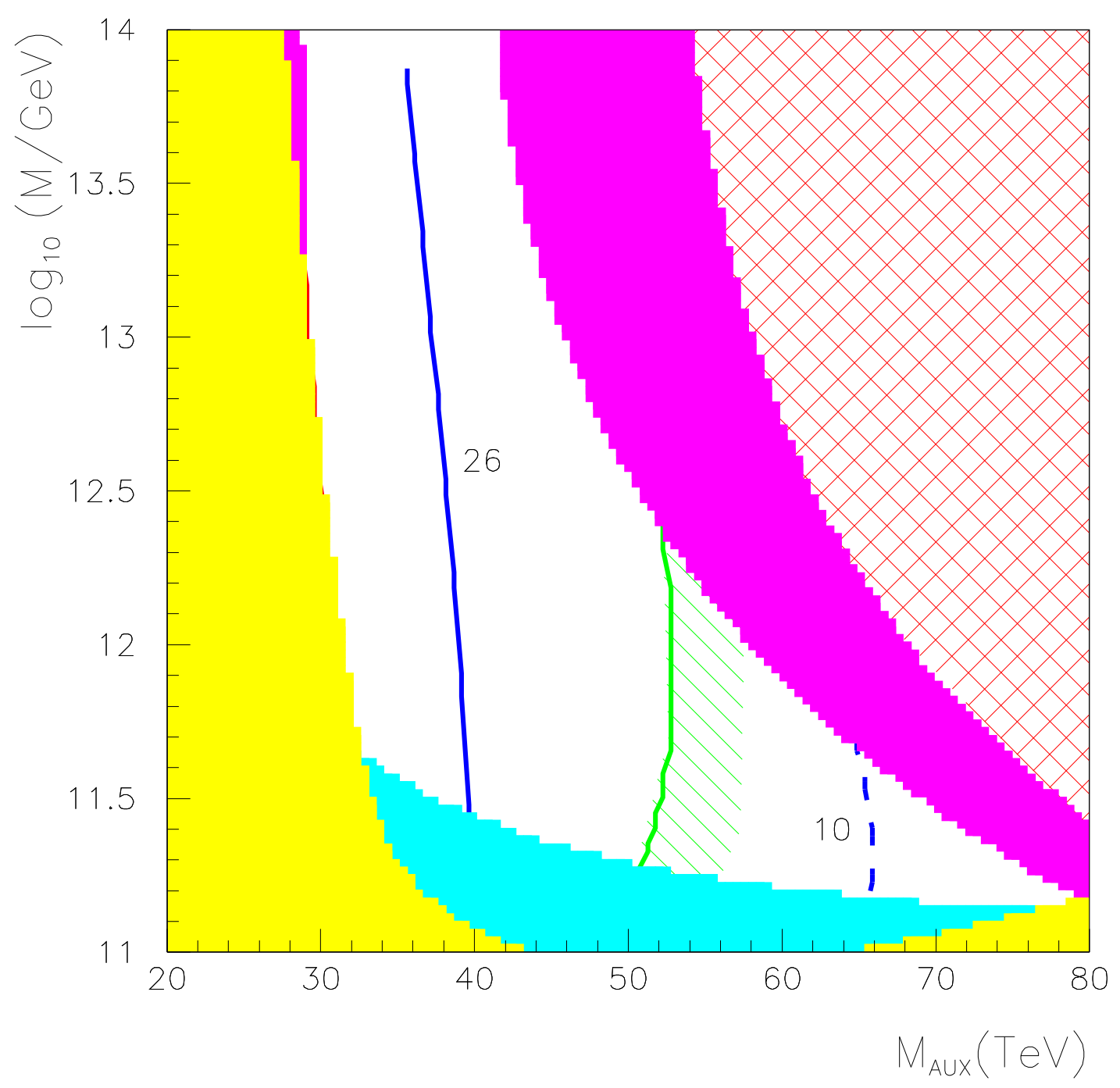

FIG. 14. Contour plot on the plane of $\left(M_{\mathrm{aux}}, M\right)$ in the deflected anomaly-mediated model with $N=6, \rho=0$ and $\tan \beta=30$. Yellow, cyan, and purple regions represent the parameter space forbidden by the lightest Higgs mass bound, the stau mass bound, and the chargino mass bound, respectively. Red cross-hashed area is the region forbidden by electroweak symmetry breaking condition. The blue solid and dashed lines stand for the $-1 \sigma$ and $-2 \sigma$ values of $a_{\mu}^{\mathrm{SUSY}}$, respectively. Green shaded area is the region allowed by $\operatorname{Br}\left(B \rightarrow X_{s} \gamma\right)$ and the green solid line corresponds to the contour of the $2 \sigma$ lower bound of $\operatorname{Br}\left(B \rightarrow X_{s} \gamma\right)$. 